\title{
CERTAIN SINGULARITIES OF POINT TRANSFORMATIONS IN SPACE OF THREE DIMENSIONS*
}

BY

S. E. URNER

1. Preliminary Definitions and Hypotheses. Let the equations

$$
x_{1}=\phi(x, y, z), \quad y_{1}=\psi(x, y, z), \quad z_{1}=\theta(x, y, z),
$$

where both sets of variables denote real, rectangular coördinates, define a transformation of the points of space. Let the functions $\phi, \psi, \theta$ be singlevalued and continuous at a point $\left(x_{0}, y_{0}, z_{0}\right)$, and let them possess, with respect to all the variables, partial derivatives of the first order, also continuous there. Then, in the neighborhood of this point, the Jacobian of the transformation,

$$
J(x, y, z) \equiv \frac{D(\phi, \psi, \theta)}{D(x, y, z)} \equiv\left|\begin{array}{ccc}
\phi_{x} & \phi_{y} & \phi_{z} \\
\psi_{x} & \psi_{y} & \psi_{z} \\
\theta_{x} & \theta_{y} & \theta_{z}
\end{array}\right|
$$

is defined and continuous. It will be assumed that $J(x, y, z)$ does not vanish identically.

In general, we may assert of the above transformation, first, that it has an inverse transformation, which shares the above-mentioned properties of the original transformation; and secondly, that it preserves the order of contact of geometrical figures (ordinary intersection being classified as contact of order zero). The further hypothesis needed to insure that the transformation possesses these properties is that, for the point under consideration, the Jacobian shall be different from zero. A point where $J=0$ is called a singular point of the transformation; in such a point we shall look for irregularities of the inverse transformation, and for peculiarities in the transformation of contact. The latter constitute, in the main, the subject-matter of the present investigation.

An economy of statement will be effected by assuming once for all that the explicit use of any derivative of $\phi, \psi$, or $\theta$ shall imply its existence and con-

\footnotetext{
* Presented to the Society February 24, 1912.
} 
tinuity at the point under consideration, as well as the possession of these properties by all the other derivatives of $\phi, \dot{\psi}$, and $\theta$ of the same and lower orders.

Under the general hypothesis that $J\left(x_{0}, y_{0}, z_{0}\right)=0$, we distinguish three cases: the matrix of the determinant used to define $J$ may be, (I), of rank 2 ; (II), of rank 1; (III), of rank 0 . We shall concern ourselves here only with Case $I, *$ so that we assume henceforth that the matrix of $J$ is of rank 2 .

2. Notations. Let $A_{1}, A_{2}, A_{3} ; B_{1}, B_{2}, B_{3}$; and $C_{1}, C_{2}, C_{3}$ denote, respectively, the cofactors of the elements of the first, second, and third rows in the Jacobian. Then let $J^{\prime}$ designate the reciprocal determinant of $J$ :

$$
J^{\prime} \equiv\left|\begin{array}{lll}
A_{1} & A_{2} & A_{3} \\
B_{1} & B_{2} & B_{3} \\
C_{1} & C_{2} & C_{3}
\end{array}\right| .
$$

Now let $A_{i}, B_{i}, C_{i}$ be a column of elements in $J^{\prime}$, not all of which vanish at the singular point in question; while $I_{1}, I_{2}, I_{3}$ constitute a row having the same property. In Case $I$ it is always possible to choose $i$ as one of the subscripts $1,2,3$, and $I$ as one of the letters $A, B, C$, so that these requirements shall be satisfied. We note that whenever the matrix of $J$ is of $\operatorname{rank} 2$, that of $J^{\prime}$ is of rank 1 . That is, at such a point,

$$
A_{1}: A_{2}: A_{3}=B_{1}: B_{2}: B_{3}=C_{1}: C_{2}: C_{3} \text {. }
$$

Whenever the ratios only are involved, the particular choice of $i$ or of $I$ is immaterial, except that, as we have provided, not all the elements in the row or column selected may vanish. We shall be dealing constantly with equalities which hold at the singular point under consideration, whose coördinates we take to be $\left(x_{0}, y_{0}, z_{0}\right)$. We shall not think it necessary always to put the quantities $x_{0}, y_{0}, z_{0}$ into evidence by writing them in place of the arguments in the functions of $x, y, z$ involved in these equations; nor to state always that the equations are to hold merely for this set of values of $x, y, z$. Equations holding for all values of $x, y, z$ will be distinguished by the use of the sign of identity. In all other cases (except where it is self-evident that something else is meant, as, for example, in the equations of curves or surfaces) the ordinary sign of equality will be used to denote equalities which hold for $x=x_{0}$, $y=y_{0}, z=z_{0}$. Where there is danger of ambiguity, we shall endeavor to avoid it by explicit statement. If a parameter $t$ is used, we shall understand that to $t=t_{0}$ correspond $x=x_{0}, y=y_{0}, z=z_{0}$, and the same rule will be followed with equations in $t$ as has been laid down for those in $x, y, z$.

* In my thesis (Ms. deposited in the Harvard University Library, 1911) I have used methods analogous to those of the present paper, in treating Cases II and III; the results there obtained, however, being much less complete than in Case I. Cf. $\$ 14$. 
Further we shall suppose that, under the transformation, to $\left(x_{0}, y_{0}, z_{0}\right)$ corresponds $\left(x_{1}^{0}, y_{1}^{0}, z_{1}^{0}\right)$.

3. Transformation of Curves-Preliminary Treatment. Let us consider the curve

$$
x=f(t), \quad y=g(t), \quad z=h(t),
$$

where $f, g, h$ are continuous, single-valued functions of the real variable $t$, which, we assume in advance, have continuous derivatives of orders 1,2 , $\cdots, k$, as a preliminary to any discussion or proposition involving derivatives of order $k$, at the point for which $t=t_{0}$. We further assume that at least one of the quantities $f^{\prime}\left(t_{0}\right), g^{\prime}\left(t_{0}\right), h^{\prime}\left(t_{0}\right)$ is different from zero. Then, if $\alpha, \beta, \gamma$ are the direction-angles of the tangent to (2) at $\left(x_{0}, y_{0}, z_{0}\right)$, we have

$$
\cos \alpha: \cos \beta: \cos \gamma=f^{\prime}\left(t_{0}\right): g^{\prime}\left(t_{0}\right): h^{\prime}\left(t_{0}\right) \text {. }
$$

The curve into which (2) is carried by the transformation is

$$
\begin{aligned}
& x_{1}=\phi[f(t), g(t), h(t)] \equiv f_{1}(t), \\
& y_{1}=\psi[f(t), g(t), h(t)] \equiv g_{1}(t), \\
& z_{1}=\theta[f(t), g(t), h(t)] \equiv h_{1}(t) .
\end{aligned}
$$

Here $f_{1}, g_{1}, h_{1}$ are single-valued and continuous, and have continuous derivatives of orders $1,2, \cdots, k$, for $t=t_{0}$, whenever $f, g, h$ are so endowed, provided $\phi, \psi, \theta$ have continuous partial derivatives of orders $1,2, \cdots, k$ at $\left(x_{0}, y_{0}, z_{0}\right)$. If now $\alpha_{1}, \beta_{1}, \gamma_{1}$ are the direction-angles of the tangent to (3) at $\left(x_{1}^{0}, y_{1}^{0}, z_{1}^{0}\right)$, then, in general,

$$
\cos \alpha_{1}: \cos \beta_{1}: \cos \gamma_{1}=f_{1}^{\prime}\left(t_{0}\right): g_{1}^{\prime}\left(t_{0}\right): h_{1}\left(t_{0}\right) \text {. }
$$

These formulæ determine the direction-cosines unless $f_{1}^{\prime}\left(t_{0}\right)=g_{1}^{\prime}\left(t_{0}\right)=$ $h_{1}^{\prime}\left(t_{0}\right)=0$. By differentiating (3) we get the formulæ

$$
\begin{aligned}
& f_{1}^{\prime}(t)=\phi_{x} f^{\prime}(t)+\phi_{y} g^{\prime}(t)+\phi_{z} h^{\prime}(t), \\
& g_{1}^{\prime}(t)=\psi_{x} f^{\prime}(t)+\psi_{y} g^{\prime}(t)+\psi_{z} h^{\prime}(t), \\
& h_{1}^{\prime}(t)=\theta_{x} f^{\prime}(t)+\theta_{y} g^{\prime}(t)+\theta_{z} h^{\prime}(t) .
\end{aligned}
$$

Then, since $J\left(x_{0}, y_{0}, z_{0}\right)$ is of rank 2 , there exists one direction for the curve (2), and only one, for which the determination (4) fails. This direction is defined by the ratios $f^{\prime}: g^{\prime}: h^{\prime}=I_{1}: I_{2}: I_{3}$. We shall call it the critical direction for the point $\left(x_{0}, y_{0}, z_{0}\right)$. Let us for the moment exclude all curves having this direction. It results from (5) that, no matter what the values of $f^{\prime}, g^{\prime}, h^{\prime}$,

$$
A_{i} f_{1}^{\prime}+B_{i} g_{1}^{\prime}+C_{i} h_{1}^{\prime}=0
$$


Therefore the tangent to the curve (3) lies in the plane through $\left(x_{1}^{0}, y_{1}^{0}, z_{1}^{0}\right)$ whose normal has the direction-ratios $A_{i}: B_{i}: C_{i}$. Let this plane be called $\Pi$. Then all curves through $\left(x_{0}, y_{0}, z_{0}\right)$, except those with the critical direction, are transformed into curves tangent to the fixed plane II .

Among the curves not having the critical direction, tangency is surely preserved by the transformation - which may, however, cause non-tangent curves to become tangent. Let us find the condition that this may happen. Suppose that $f_{1}^{\prime}, g_{1}^{\prime}, h_{1}^{\prime}$ have certain fixed values which satisfy (6). Then the matrix

$$
\left\|\begin{array}{llll}
\phi_{x} & \phi_{y} & \phi_{z} & f_{1}^{\prime} \\
\psi_{x} & \psi_{y} & \psi_{z} & g_{1}^{\prime} \\
\theta_{x} & \theta_{y} & \theta_{z} & h_{1}^{\prime}
\end{array}\right\|
$$

is of rank 2. Therefore there will be at least one pair of the equations (5), (considered as equations in $f^{\prime}, g^{\prime}, h^{\prime}$ ) which are independent, and whose common solutions satisfy the third. Suppose, for example, that the first two equations are independent. Then $f_{1}^{\prime}$ and $g_{1}^{\prime}$ are not both zero, for otherwise, by $(6), h_{1}^{\prime}=0$. We note at once that

$$
g_{1}^{\prime}\left(\phi_{x} f^{\prime}+\phi_{y} g^{\prime}+\phi_{z} h^{\prime}\right)-f_{1}^{\prime}\left(\psi_{x} f^{\prime}+\psi_{y} g^{\prime}+\psi_{z} h^{\prime}\right)=0 .
$$

On the other hand, if $f^{\prime}, g^{\prime}, h^{\prime}$ are given any values (distinct from the critical values) satisfying (7), then

$$
\begin{aligned}
& \phi_{x} f^{\prime}+\phi_{y} g^{\prime}+\phi_{z} h^{\prime}=k f_{1}^{\prime}, \\
& \psi_{x} f^{\prime}+\psi_{y} g^{\prime}+\psi_{z} h^{\prime}=k g_{1}^{\prime}
\end{aligned}
$$

That is, (7) is the necessary and sufficient condition upon $f^{\prime}, g^{\prime}, h^{\prime}$, that the curve (3) shall be transformed into a curve having the given direction $f_{1}^{\prime}: g_{1}^{\prime}: h_{1}^{\prime}$. We note, moreover, that for all values of $f_{1}^{\prime}$ and $g_{1}^{\prime}$, (7) is satisfied if $f^{\prime}: g^{\prime}: h^{\prime}=I_{1}: I_{2}: I_{3}$. Since (7) is a linear, homogeneous equation in $f^{\prime}, g^{\prime}, h^{\prime}$, the tangent to (2) lies in a certain fixed plane through the line having the critical direction. Hence the result,

THEOREM 1. The necessary and sufficient condition that two non-tangent curves through $\left(x_{0}, y_{0}, z_{0}\right)$, which have there directions distinct from the critical direction, be rendered tangent by the transformation, is that the plane of their tangents at the point contain the line having the critical direction.

4. Continuation: Curves with Critical Direction. Let us now assume that the curve (2) has the critical direction. Suppose, however, that not all the quantities $f_{1}^{\prime \prime}\left(t_{0}\right), g_{1}^{\prime \prime}\left(t_{0}\right), h_{1}^{\prime \prime}\left(t_{0}\right)$ are zero. Then

$$
f_{1}^{\prime}(t) \equiv f_{1}^{\prime \prime}\left[t_{0}+\theta_{1}\left(t-t_{0}\right)\right]\left(t-t_{0}\right) \quad\left(0<\theta_{1}<1\right) .
$$


with similar formulæ for $g_{1}^{\prime}, h_{1}^{\prime}$. Hence

$f_{1}^{\prime}(t): g_{1}^{\prime}(t): h_{1}^{\prime}(t) \equiv f_{1}^{\prime \prime}\left[t_{0}+\theta_{1}\left(t-t_{0}\right)\right]: g_{1}^{\prime \prime}\left[t_{0}+\theta_{2}\left(t-t_{0}\right)\right]:$

Allowing $t$ to approach $t_{0}$, we see that

$$
h_{1}^{\prime \prime}\left[t_{0}+\theta_{8}\left(t-t_{0}\right)\right] \text {. }
$$

$$
\cos \alpha_{1}: \cos \beta_{1}: \cos \gamma_{1}=f_{1}^{\prime \prime}\left(t_{0}\right): g_{1}^{\prime \prime}\left(t_{0}\right): h_{1}^{\prime \prime}\left(t_{0}\right) \text {. }
$$

Placing $f^{\prime}=k I_{1}, g^{\prime}=k I_{2}, h^{\prime}=k I_{3}$, we have

where

$$
\begin{aligned}
& f_{1}^{\prime \prime}=\phi_{x} f^{\prime \prime}+\phi_{y} g^{\prime \prime}+\phi_{z} h^{\prime \prime}+k^{2} U^{2}(\phi, I), \\
& g_{1}^{\prime \prime}=\psi_{x} f^{\prime \prime}+\psi_{y} g^{\prime \prime}+\psi_{z} h^{\prime \prime}+k^{2} U^{2}(\psi, I), \\
& h_{1}^{\prime \prime}=\theta_{x} f^{\prime \prime}+\theta_{y} g^{\prime \prime}+\theta_{z} h^{\prime \prime}+k^{2} U^{2}(\theta, I),
\end{aligned}
$$

$U^{2}(F, I) \equiv I_{1}^{2} F_{x x}+I_{2}^{2} F_{y y}+I_{3}^{2} F_{z z}+2 I_{2} I_{3} F_{y z}+2 I_{3} I_{1} F_{z x}+2 I_{1} I_{2} F_{x y}$.

If we make a change of parameter, $t^{\prime}=k t$, the functions $f, g, h$ go over into such functions $\bar{f}, \bar{g}, \bar{h}$ of $t^{\prime}$ that, when $t^{\prime}=k t_{0}, \bar{f}^{\prime}=I_{1}, \bar{g}^{\prime}=I_{2}, \bar{h}^{\prime}=I_{3}$. Then let us drop dash and prime, merely supposing the necessary changes already made in the functions $f, g, h$ as they stand. This done, we have a right to set $k$ in equations (9) equal to unity.

But we conclude from (9) that

$$
A_{i} f_{1}^{\prime \prime}+B_{i} g_{1}^{\prime \prime}+C_{i} h_{1}^{\prime \prime}=P,
$$

where

$$
P=A_{i} U^{2}(\phi, I)+B_{i} U^{2}(\psi, I)+C_{i} U^{2}(\theta, I) \text {. }
$$

First, suppose $P \neq 0$. Then it is clear that no curve having the critical direction can become a curve tangent to $\Pi$; for the value of the quantity $P$ is independent of the particular curve (2). Aside from the fact that it does not lie in $I I$, no restriction is imposed by (10) upon the direction of the tangent to the transformed curve. For, if we conceive of $f_{1}^{\prime \prime}, g_{1}^{\prime \prime}, h_{1}^{\prime \prime}$ as given numbers, satisfying (10), it is possible to find values of $f^{\prime \prime}, g^{\prime \prime}, h^{\prime \prime}$ to satisfy (9). On account of (10), the matrix of the coefficients in (9) is of rank 2 . Since the matrix of $J$ is of rank 2 , there are two of these equations which are consistent and independent, and whose common solutions satisfy the third. There will be a one-parameter family of these solutions.

On the other hand, if $f^{\prime \prime}, g^{\prime \prime}, h^{\prime \prime}$ have any values which satisfy the relations

$$
\begin{aligned}
I_{3} g^{\prime \prime}-I_{2} h^{\prime \prime} & =p, \\
-I_{3} f^{\prime \prime}+I_{1} h^{\prime \prime} & =q, \\
I_{2} f^{\prime \prime}-I_{1} g^{\prime \prime} & =r,
\end{aligned}
$$


$p, q, r$ being any numbers such that $I_{1} p+I_{2} q+I_{3} r=0$, their substitution in (9) will yield certain fixed values for $f_{1}^{\prime \prime}, g_{1}^{\prime \prime}, h_{1}^{\prime \prime}$; and these values will satisfy (10). This results from elimination of $f^{\prime \prime}, g^{\prime \prime}, h^{\prime \prime}$ between (9) and any two of (11) which are independent. Conversely, the one-parameter set of values of $f^{\prime \prime}, g^{\prime \prime}, h^{\prime \prime}$ which correspond to fixed values of $f_{1}^{\prime \prime}, g_{1}^{\prime \prime}, h_{1}^{\prime \prime}$ will satisfy (11) for some admissible set of values of $p, q, r$. To prove this, we need only eliminate $f^{\prime \prime}, g^{\prime \prime}, h^{\prime \prime}$ successively between two of equations (9) which are independent. But (11) will be recognized as the conditions that the curve (2) have contact of at least the second order with some fixed curve. Hence,

Theorem 2. The necessary and sufficient condition that two curves having the critical direction be transformed into tangent curves is that they have contact of at least the second order.

5. Conditions for Complanar Tangents. To speak roughly, then, the noncritical directions, constituting a two-parameter family, correspond to the one-parameter family of directions in the plane $\Pi$, each plane' of directions through the critical tangent being compressed into a single direction; whereas, the critical direction itself explodes into the whole two-parameter family of directions at $\left(x_{1}^{0}, y_{1}^{0}, z_{1}^{0}\right)$, exclusive of those in $\Pi$. It will be useful, however, to inquire more closely into this latter correspondence. Our first step is as follows:

Theorem 3. Any family of curves through $\left(x_{0}, y_{0}, z_{0}\right)$. which have there the critical direction, and which also have in that point the same osculating plane, will be transformed into a family of curves whose tangents at $\left(x_{1}^{0}, y_{1}^{0}, z_{1}^{0}\right)$ are complanar. Each plane of tangents thus determined will pass through the line whose direction-ratios are $U^{2}(\phi, I): U^{2}(\psi, I): U^{2}(\theta, I)$. Conversely, if the common tangent plane to two of the transformed curves contains this line, then the original curves have the same osculating plane at $\left(x_{0}, y_{0}, z_{0}\right)$.

Among the curves with the critical direction, those will have the same osculating plane, and only those, for which

$$
a f^{\prime \prime}\left(t_{0}\right)+b g^{\prime \prime}\left(t_{0}\right)+c h^{\prime \prime}\left(t_{0}\right)=0,
$$

$a, b, c$ being constants, such that $a I_{1}+b I_{2}+c I_{3}=0, a^{2}+b^{2}+c^{2} \neq 0$. For the osculating plane of such a curve at $\left(x_{0}, y_{0}, z_{0}\right)$ will have the equation

$$
\begin{aligned}
& \left(h^{\prime \prime} I_{2}-g^{\prime \prime} I_{3}\right)\left(x-x_{0}\right)+\left(f^{\prime \prime} I_{3}-h^{\prime \prime} I_{1}\right)\left(y-y_{0}\right) \\
& +\left(g^{\prime \prime} I_{1}-f^{\prime \prime} I_{2}\right)\left(z-z_{0}\right)=0 .
\end{aligned}
$$

If we suppose (12) satisfied, then the three coefficients in (13) will have values proportional to $a, b, c$. On the other hand, it is easy to see that when the coefficients in (13) have ratios $a: b: c$ independent of $f^{\prime \prime}, g^{\prime \prime}, h^{\prime \prime}$, then these latter quantities satisfy (12). 
But if we adjoin the condition (12) to (9), there results a linear relation between $f_{1}^{\prime \prime}, g_{1}^{\prime \prime}, h_{1}^{\prime \prime}$. In fact, if, for example, $A_{1} \neq 0$, then we have

$$
\begin{array}{ll}
b & \theta_{y} \\
c & \theta_{z}
\end{array}\left(g_{1}^{\prime \prime}-U^{2}(\psi, I)\right)-\left|\begin{array}{cc}
b & \psi_{y} \\
c & \psi_{z}
\end{array}\right|\left(h_{1}^{\prime \prime}-U^{2}(\theta, I)\right)=0 \text {, }
$$

this relation being independent of (10), since $A_{1} \neq 0$. Now (10) and (14) are to be considered as equivalent to a single homogeneous linear condition upon $f_{1}^{\prime \prime}, g_{1}^{\prime \prime}, h_{1}^{\prime \prime}$, which of course confines the tangent of the transformed curve to a fixed plane. Further, both (10) and (14) are satisfied, for all values of $a, b, c$, if

$$
f_{1}^{\prime \prime}=U^{2}(\phi, I), \quad g_{1}^{\prime \prime}=U^{2}(\psi, I), h_{1}^{\prime \prime}=U^{2}(\theta, I) .
$$

If now we subject $f_{1}^{\prime \prime}, g_{1}^{\prime \prime}, h_{1}^{\prime \prime}$, to any condition of the form

$$
\lambda\left(f_{1}^{\prime \prime}-U^{2}(\phi, I)\right)+\mu\left(g_{1}^{\prime \prime}-U^{2}(\psi, I)\right)+\nu\left(h_{1}^{\prime \prime}-U^{2}(\theta, I)\right)=0,
$$

where $\lambda^{2}+\mu^{2}+\nu^{2} \neq 0$, in addition to $(10)$, then $f^{\prime \prime}, g^{\prime \prime}, h^{\prime \prime}$ satisfy a relation like (12). To show this, we need only substitute in (15) the values of $f_{1}^{\prime \prime}, g_{1}^{\prime \prime}$, $h_{1}^{\prime \prime}$ from (9), thus obtaining

$\left(\lambda \phi_{x}+\mu \psi_{x}+\nu \theta_{x}\right) f^{\prime \prime}+\left(\lambda \phi_{y}+\mu \psi_{y}+\nu \theta_{y}\right) g^{\prime \prime}+\left(\lambda \phi_{z}+\mu \psi_{z}+\nu \theta_{z}\right) h^{\prime \prime}=0$.

The coefficients of $f^{\prime \prime}, g^{\prime \prime}, h^{\prime \prime}$ here evidently satisfy the conditions placed upon $a, b, c$. They are not all zero, since we are not at present dealing with the plane $\Pi$. Theorem 3 is therefore proved.

It is to be noted that when

$$
h^{\prime \prime} I_{2}-g^{\prime \prime} I_{3}=f^{\prime \prime} I_{3}-h^{\prime \prime} I_{1}=g^{\prime \prime} I_{1}-f^{\prime \prime} I_{2}=0,
$$

the equation of the osculating plane, (13), disappears, and our theorem becomes illusory. All curves for which this happens have contact of the second order with each of their tangent planes and with the line having the critical direction. The transformed curves are tangent to the transform of this straight line, which at $\left(x_{1}^{0}, y_{1}^{0}, z_{1}^{0}\right)$ has the direction

$$
U^{2}(\phi, I): U^{2}(\psi, I): U^{2}(\theta, I) \text {. }
$$

The preceding theorem is, however, a very special one. It shows how a family of curves which osculate a certain plane are carried into curves tangent to a certain plane-the latter always containing a fixed line. It will be useful to generalize this, and to find what condition a curve must fulfill in order that its transformed curve may be tangent to a specified plane.

For this purpose, let us consider the equations (9) (with $k=1$ ). If the tangent to the transformed curve lies in the plane whose normal has the direction-ratios $\lambda: \mu: \nu$, then

$$
\lambda f_{1}^{\prime \prime}+\mu g_{1}^{\prime \prime}+\nu h_{1}^{\prime \prime}=0 \text {. }
$$


The corresponding condition imposed by (9) upon $f^{\prime \prime}, g^{\prime \prime}, h^{\prime \prime}$ is

$$
\begin{aligned}
& \left(\lambda \phi_{x}+\mu \psi_{x}+\nu \theta_{x}\right) f^{\prime \prime}+\left(\lambda \phi_{y}+\mu \psi_{\nu}+\nu \theta_{\nu}\right) g^{\prime \prime} \\
& +\left(\lambda \phi_{z}+\mu \psi_{z}+\nu \theta_{z}\right) h^{\prime \prime}+\lambda U^{2}(\phi, I)+\mu U^{2}(\psi, I)+\nu U^{2}(\theta, I)=0 .
\end{aligned}
$$

Moreover, if we construct the relation (16) for an arbitrary set of values of $\lambda, \mu, \nu$, then all the curves satisfying it will go over into curves normal at $\left(x_{1}^{0}, y_{1}^{0}, z_{1}^{0}\right)$ to the line with direction-ratios $\lambda: \mu: \nu$. It will be observed, however, that if we multiply the coefficients of $f^{\prime \prime}, g^{\prime \prime}, h^{\prime \prime}$ in (16) by $I_{1}, I_{2}, I_{3}$, respectively, and add, the result is zero; and conversely, any equation of the form

$$
\xi f^{\prime \prime}+\eta g^{\prime \prime}+\zeta h^{\prime \prime}+\omega=0
$$

where

$$
\xi I_{1}+\eta I_{2}+\zeta I_{3}=0, \text { but } \xi^{2}+\eta^{2}+\zeta^{2} \neq 0,
$$

is equivalent to a relation of the form (16), $\lambda, \mu, \nu$ being properly chosen. For, if we set,

$$
\begin{gathered}
\lambda \phi_{x}+\mu \psi_{x}+\nu \theta_{x}=\xi, \\
\lambda \phi_{y}+\mu \psi_{\nu}+\nu \theta_{y}=\eta, \\
\lambda \phi_{z}+\mu \psi_{z}+\nu \theta_{z}=\zeta, \\
\lambda U^{2}(\phi, I)+\mu U^{2}(\psi, I)+\nu U^{2}(\theta, I)=\omega,
\end{gathered}
$$

then we can solve uniquely for $\lambda, \mu, \nu$. In fact, the first three of these equations are connected by a linear relation, since $J=0$, and

$$
I_{1} \xi+I_{2} \eta+I_{3} \zeta=0
$$

but there will always be two of them which are independent, since $J$ is of rank two. Further, the fourth equation is independent of, and consistent with, the others, since we have supposed that

$$
P=A_{i} U^{2}(\phi, I)+B_{i} U^{2}(\psi, I)+C_{i} U^{2}(\theta, I) \neq 0 .
$$

We have now indicated sufficiently how to find the plane of directions which corresponds to a given relation (17).

But it is possible to attach to the condition (17) a more concrete significance. If the surface $F(x, y, z)=0$ and the curve

$$
x=f(t), \quad y=g(t), \quad z=h(t)
$$

pass through $\left(x_{0}, y_{0}, z_{0}\right)$, corresponding to $t=t_{0}$, the conditions for contact of at least the second order between them at this point are, that for $t=t_{0}$,

$$
\frac{d F[f(t), g(t), h(t)]}{d t}=\frac{d^{2} F[f(t), g(t), h(t)]}{d t^{2}}=0 .
$$


That is, and,

$$
F_{x} f^{\prime}+F_{y} g^{\prime}+F_{z} h^{\prime}=0
$$

where

$$
U^{2}\left(F, f^{\prime}, g^{\prime}, h^{\prime}\right)=F_{x x} f^{\prime 2}+F_{y y} g^{\prime 2}+F_{z z} h^{\prime 2}+2 F_{y z} g^{\prime} h^{\prime}+2 F_{z x} h^{\prime} f^{\prime}+2 F_{x y} f^{\prime} g^{\prime} .
$$

If the surface is tangent to the curves having the critical direction, so that (19) is fulfilled, then it follows that (20) is of the form (17); that is,

Therefore,

$$
F_{x} I_{1}+F_{y} I_{2}+F_{z} I_{3}=0
$$

THEOREM 4. A family of curves through $\left(x_{0}, y_{0}, z_{0}\right)$ which have there the critical direction, and which have in that point contact of at least the second order with the same surface, will be transformed into a family of curves whose tangents at $\left(x_{1}^{0}, y_{1}^{0}, z_{1}^{0}\right)$ are complanar. Conversely, if a family of curves having the critical direction are transformed into curves with complanar tangents, there will exist a surface with which they all have contact of at least the second order.

6. Singularity of the Second Order. We have assumed thus far that $P \neq 0$, and also that $f_{1}^{\prime \prime}, g_{1}^{\prime \prime}, h_{1}^{\prime \prime}$ are not all zero. The first assumption made, the second is superfluous. For $P=0$ is precisely the condition that the equations obtained by setting, in (9), $f_{1}^{\prime \prime}=g_{1}^{\prime \prime}=h_{1}^{\prime \prime}=0$, be consistent. That is, if $P \neq 0$, the directions of the transformed curves depend upon derivatives of the first and second orders only. This being true, we shall say that the point $\left(x_{0}, y_{0}, z_{0}\right)$ is a singular point of the first order. On the other hand, if it happens that $P=0$, then the order of the singularity will be higher; and if the directions of all the transformed curves can be found by means of derivatives of the first, second, and third orders, the point shall be a singular point of the second order.

The detailed description of the properties of the singular point of the second order will be omitted, since they may very well be included in the discussion of the general case. We merely stop to note that, when $P=0$, we are assured by equation (10) that all curves having the critical direction, but for which $f^{\prime \prime}, g^{\prime \prime}, h^{\prime \prime}$ do not have the critical values needed to make $f_{1}^{\prime \prime}=g_{1}^{\prime \prime}=h_{1}^{\prime \prime}=0$, are transformed into curves tangent to $\Pi$. They are, in a manner, superseded in the enjoyment of singular properties, by those curves whose equations yield critical values of the second derivatives also. These latter curves we shall expect to play the same rôle as was played in the earlier case by curves having the critical direction.

But it will be a matter of some importance to develop a practical test for determining whether or not a given point is a singular point of the second 
order. The construction of the quantity $P$ is a rather laborious process, which, moreover, does not readily admit of extension to the higher cases. For this purpose, let us make use of the following identity in $x, y, z$ :

$$
\begin{aligned}
A_{1} J_{x} & +A_{2} J_{y}+A_{3} J_{z} \equiv A_{1}^{2} \phi_{x x}+A_{2}^{2} \phi_{y y}+A_{3}^{2} \phi_{x z}+2 A_{2} A_{3} \phi_{y z}+2 A_{3} A_{1} \phi_{x x} \\
& +2 A_{1} A_{2} \phi_{x y}+A_{1} B_{1} \psi_{x x}+A_{2} B_{2} \psi_{y y}+A_{3} B_{3} \psi_{z z}+\left(A_{2} B_{3}+A_{3} B_{2}\right) \psi_{y z} \\
& +\left(A_{1} B_{3}+A_{3} B_{1}\right) \psi_{z x}+\left(A_{1} B_{2}+A_{2} B_{1}\right) \psi_{x y}+A_{1} C_{1} \theta_{x x}+A_{2} C_{2} \theta_{y y} \\
& +A_{3} C_{3} \theta_{x z}+\left(A_{2} C_{3}+A_{3} C_{2}\right) \theta_{y z}+\left(A_{1} C_{3}+A_{3} C_{1}\right) \theta_{x x}+\left(A_{1} C_{2}+A_{2} C_{1}\right) \theta_{x y} .
\end{aligned}
$$

From the fact that $J\left(x_{0}, y_{0}, z_{0}\right)=0$, certain reductions take place in the right member when we put $x_{0}, y_{0}, z_{0}$ for $x, y, z$; for then, $A_{1}: A_{2}: A_{3}=B_{1}: B_{2}: B_{3}=C_{1}: C_{2}: C_{3}$. Let us suppose, to show how this reduction comes about, that $A_{1} \neq 0$. Then the right member above becomes,

$$
\begin{aligned}
A_{1}^{2} \phi_{x x} & +A_{2}^{2} \phi_{y y}+A_{3}^{2} \phi_{x z}+2 A_{2} A_{3} \phi_{y z}+2 A_{3} A_{1} \phi_{x x}+2 A_{1} A_{2} \phi_{x y} \\
& +\frac{B_{1}}{A_{1}}\left[A_{1}^{2} \psi_{x x}+2 A_{1} A_{2} \psi_{x y}+2 A_{1} A_{3} \psi_{x z}\right]+\frac{B_{2}}{A_{2}}\left[A_{2}^{2} \psi_{y y}+2 A_{2} A_{3} \psi_{y z}\right] \\
& +\frac{B_{3}}{A_{3}} A_{3}^{2} \psi_{z x}+\frac{C_{1}}{A_{1}}\left[A_{1}^{2} \theta_{x x}+2 A_{1} A_{2} \theta_{x y}+2 A_{1} A_{3} \theta_{x z}\right] \\
& +\frac{C_{2}}{A_{2}}\left[A_{2}^{2} \theta_{y y}+2 A_{2} A_{3} \theta_{y z}\right]+\frac{C_{3}}{A_{3}} A_{3}^{2} \theta_{z x} .
\end{aligned}
$$

Here, if it happens that $A_{2}=0$, we do not need to trouble ourselves about the ratio $B_{2} / A_{2}$, for the terms which give rise to it will not occur at all, and in such case we will suppose them stricken out. A similar remark applies to $A_{3}$. But if $A_{2}$ actually occurs, we may replace $B_{2} / A_{2}$ by $B_{1} / A_{1}$, and if $A_{3}$ occurs, we may replace $B_{3} / A_{3}$ by $B_{1} / A_{1}$. Likewise, $C_{2} / A_{2}=C_{3} / A_{3}=C_{1} / A_{1}$. This done, we have at once,

(21) $A_{1}\left(A_{1} J_{x}+A_{2} J_{y}+A_{3} J_{z}\right)=A_{1} U^{2}(\phi, A)+B_{1} U^{2}(\psi, A)+C_{1} U^{2}(\theta, A)$.

This case is entirely typical, and we can say in general, that if $I_{i} \neq 0$,

(22) $I_{i}\left(I_{1} J_{x}+I_{2} J_{y}+I_{3} J_{z}\right)=A_{i} U^{2}(\phi, I)+B_{i} U^{2}(\psi, I)+C_{i} U^{2}(\theta, I)$,

for $I=A, B, C$, and $i=1,2,3$. Therefore, the condition $P=0$, that is, the condition for a singularity of the second order, is exactly equivalent to the condition,

$$
I_{1} J_{x}+I_{2} J_{y}+I_{3} J_{z}=0 \text {. }
$$

The equation (23) is in reality equivalent to the three equations

$$
\frac{D(\phi, \psi, J)}{D(x, y, z)}=0 ; \quad \frac{D(\psi, \theta, J)}{D(x, y, z)}=0 ; \quad \frac{D(\theta, \phi, J)}{D(x, y, z)}=0 .
$$


There will be at least one of these which is not trivial by reason of the vanishing of all the coefficients of the derivatives of $J$. Denote the left member of this equation, before the values $x_{0}, y_{0}, z_{0}$ have been substituted, by $J_{1}(x, y, z)$. (It may be possible to choose $J_{1}$ in more than one way, but the precise choice is immaterial, since the values of any two of them at $\left(x_{0}, y_{0}, z_{0}\right)$ differ by a factor different from zero). This done, we may state our result as follows:

Theorem 5. The necessary and sufficient conditions that $\left(x_{0}, y_{0}, z_{0}\right)$ be a singular point of at least the second order are that

$$
J\left(x_{0}, y_{0}, z_{0}\right)=0, \quad \text { and } \quad J_{1}\left(x_{0}, y_{0}, z_{0}\right)=0 .
$$

7. Normalization of Parameter. In the further treatment of the curves having the critical direction, we shall find it of advantage to take the equations of any curve in a certain canonical form. A first step in this direction was taken in $\$ 4$, when we agreed to make such a change of parameter that, for every curve with the critical direction, $f^{\prime}=I_{1}, g^{\prime}=I_{2}, h^{\prime}=I_{3}$. What we desire to do now, is to extend this normalization to the derivatives of any order with which we may be concerned. For this purpose, let us assume that by some means the normalization has been effected for the derivatives of orders $1,2, \cdots, k-1$, in such a manner that the necessary and sufficient conditions for contact of order $n$ between two of the curves in question will be that their equations give rise to the same values of the derivatives $f^{(j)}, g^{(j)}, h^{(j)}$, for $j=1,2, \cdots, n$, and for every value of $n$ from 1 to $k-1$.

Then the derivatives of order $k$ taken from the equations of any curve of the family which has contact of order $k$, at least, with a specified curve $C$ of the family, will satisfy the relations,

$$
\begin{aligned}
I_{3} g^{(k)}-I_{2} h^{(k)} & =p, \\
-I_{3} f^{(k)}+I_{1} h^{(k)} & =q, \\
I_{2} f^{(k)}-I_{1} g^{(k)} & =r,
\end{aligned}
$$

where $p, q, r$ depend only on the choice of $C$, and $I_{1} p+I_{2} q+I_{3} r=0$.

We may now normalize the derivatives of order $k$ by subjecting them to a condition of the form

$$
\alpha f^{(k)}+\beta g^{(k)}+\gamma h^{(k)}=\delta,
$$

provided (26) is always independent of, and consistent with, the equations (25); for then the effect of (26) will be to admit one, and only one, set of values of $f^{(k)}, g^{(k)}, h^{(k)}$ from the one-parameter family of solutions of (25). It will result from this, that the necessary and sufficient conditions for contact of order $k$, at least, between two curves of the family under consideration, will 
be that the $k$ th order derivatives, taken from their equations, have the same values for $t=t_{0}$. Now (26) is always independent of, and consistent with, the equations (25), if, and only if,

$$
I_{1} \alpha+I_{2} \beta+I_{3} \gamma \neq 0 .
$$

Under all circumstances, then, we may put $\alpha=I_{1}, \beta=I_{2}, \gamma=I_{3}$. The value of $\delta$ is immaterial, but let us call it $\delta_{k}$. It is subject to no restriction except that $\delta_{1}=I_{1}^{2}+I_{2}^{2}+I_{3}^{2}$. If $\delta_{k} \neq 0$, the values $f^{(k)}=g^{(k)}=h^{(k)}=0$ are excluded, but we shall agree to include them in the family

$$
\left.f^{(k)}=\lambda I_{1}, \quad g^{(k)}=\lambda I_{2}, \quad h^{(k)}=\lambda I_{3} \quad \text { ( } \lambda \text { a parameter }\right) .
$$

If now in the above we set $k=2$, then the conditions are satisfied which enable us to appy it when $k=3$, and so on. So that the desired result - the the normalization of the derivatives of orders $1,2, \cdots, n$ - will be effected by requiring that

$$
I_{1} f^{(j)}+I_{2} g^{(j)}+I_{3} h^{(j)}=\delta_{j} \quad(j=1,2, \cdots, n) .
$$

It may now be inquired whether the $n$ conditions thus imposed can all be satisfied for an arbitrary curve with the critical direction. Let us suppose that

$$
x=F(\lambda), \quad y=G(\lambda), \quad z=H(\lambda)
$$

is any curve such that

where

$$
F^{\prime}\left(\lambda_{0}\right): G^{\prime}\left(\lambda_{0}\right): H^{\prime}\left(\lambda_{0}\right)=I_{1}: I_{2}: I_{3},
$$

$$
x_{0}=F\left(\lambda_{0}\right), \quad y_{0}=G\left(\lambda_{0}\right), \quad z_{0}=H\left(\lambda_{0}\right) .
$$

By means of the substitution

$$
\lambda \equiv \alpha(t) \equiv \lambda_{0}+\sum_{k=1}^{k=n} \frac{\alpha_{k}}{k !}\left(t-t_{0}\right)^{k},
$$

let the functions $F, G, H$ become $f(t), g(t), h(t)$. Then it is possible to choose the coefficients $\alpha_{k}$ in such a manner that the equations (27) are all satisfied for $t=t_{0}$. In the first place,

$$
\frac{d f(t)}{d t}=\frac{d F(\lambda)}{d \lambda} \frac{d \lambda}{d t}
$$

and when $t=t_{0}, f^{\prime}=F^{\prime} \alpha_{1}$. Similarly, $g^{\prime}=G^{\prime} \alpha_{1}$ and $h^{\prime}=H^{\prime} \alpha_{1}$. Then put

Let

$$
I_{1} f^{\prime}+I_{2} g^{\prime}+I_{3} h^{\prime}=\alpha_{1}\left(I_{1} F^{\prime}+I_{2} G^{\prime}+I_{3} H^{\prime}\right)=\delta_{1} .
$$

$$
I_{1} F^{\prime}+I_{2} G^{\prime}+I_{3} H^{\prime}=\gamma_{1} .
$$

Then surely $\gamma_{1} \neq 0$; for $F^{\prime}=k I_{1}, G^{\prime}=k I_{2}, H^{\prime}=k I_{3}$, and $k \neq 0$. That is, $\alpha_{1}=\delta_{1} / \gamma_{1}$. 
Further,

$$
\frac{d^{2} f(t)}{d t^{2}} \equiv \frac{d}{d t}\left[\frac{d F}{d \lambda} \frac{d \lambda}{d t}\right] \equiv \frac{d F}{d \lambda} \frac{d^{2} \lambda}{d t^{2}}+\frac{d^{2} F}{d \lambda^{2}}\left(\frac{d \lambda}{d t}\right)^{2},
$$

or, when $t=t_{0}$,

$$
f^{\prime \prime}=F^{\prime} \alpha_{2}+F^{\prime \prime} \alpha_{1}^{2} \text {. }
$$

Likewise, $g^{\prime \prime}=G^{\prime} \alpha_{2}+G^{\prime \prime} \alpha_{1}^{2}$ and $h^{\prime \prime}=H^{\prime} \alpha_{2}+H^{\prime \prime} \alpha_{1}^{2}$. Then

where

$$
I_{1} f^{\prime \prime}+I_{2} g^{\prime \prime}+I_{3} h^{\prime \prime}=\alpha_{2} \gamma_{1}+\alpha_{1}^{2} \gamma_{2}=\delta_{2} \text {, }
$$

Then we have,

$$
\gamma_{2}=I_{1} F^{\prime \prime}+I_{2} G^{\prime \prime}+I_{3} H^{\prime \prime} \text {. }
$$

$$
\alpha_{2}=\frac{\delta_{2}-\alpha_{1}^{2} \gamma_{2}}{\gamma_{1}}=\frac{\gamma_{1}^{2} \delta_{2}-\delta_{1}^{2} \gamma_{2}}{\gamma_{1}^{3}} \text {. }
$$

Obviously, this process can be continued as far as we like. Each new condition introduces just one new coefficient, which is itself affected by the coefficient $\gamma_{1}$. There will therefore arise neither indetermination nor impossibility, no matter how far we choose to carry the process. The coefficients $\alpha_{k}$ being thus determined, it is clear that equations (27) are satisfied. We shall, hereafter, assume the reduction just described to have been made in the equations of all curves considered.

8. The General Case. Let us now suppose that there exists a curve through $\left(x_{0}, y_{0}, z_{0}\right)$ for which $f^{(j)}, g^{(j)}, h^{(j)}$ have such values that

$$
f_{1}^{(j)}\left(t_{0}\right)=g_{1}^{(j)}\left(t_{0}\right)=h_{1}^{(j)}\left(t_{0}\right)=0,
$$

for $j=1,2, \cdots, k-1$, but $\left[f_{1}^{(k)}\right]^{2}+\left[g_{1}^{(k)}\right]^{2}+\left[h_{1}^{(k)}\right]^{2} \neq 0$. Then let us join the point $t=t_{0}$ on the transformed curve with the point $\left(x_{1}, y_{1}, z_{1}\right)$, for which $t=t_{0}+\epsilon$, by means of a secant. The direction-ratios of this line will be $x_{1}-x_{1}^{0}: y_{1}-y_{1}^{0}: z_{1}-z_{1}^{0}$, where

$$
x_{1}-x_{1}^{0}=f_{1}\left(t_{0}+\epsilon\right)-f_{1}\left(t_{0}\right)=\frac{\epsilon^{k}}{k !} f_{1}^{(k)}\left[t_{0}+\theta_{1} \epsilon\right] \quad\left(0<\theta_{1}<1\right),
$$

with similar formulæ for $y_{1}-y_{1}^{0}$ and $z_{1}-z_{1}^{0}$. Now if we allow $\epsilon$ to approach zero, we obtain for the direction-ratios of the tangent to the curve,

$$
\cos \alpha_{1}: \cos \beta_{1}: \cos \gamma_{1}=f_{1}^{(k)}\left(t_{0}\right): g_{1}^{(k)}\left(t_{0}\right): h_{1}^{(k)}\left(t_{0}\right) \text {. }
$$

Let us now make clear the notion of the order of a singular point. If there exist curves satisfying the requirements of the preceding paragraph, then we shall say that $\left(x_{0}, y_{0}, z_{0}\right)$ is a singular point of order $k-1$, at least. The order will be exactly $k-1$, if there is no curve for which

$$
f_{1}^{(j)}=g_{1}^{(j)}=h_{1}^{(j)}=0, \text { for } j=1,2 ; \cdots, k .
$$

That is, the order of the singularity is precisely one unit less than the order 
of the highest derivatives which must be used to find the direction of the transform of every curve through $\left(x_{0}, y_{0}, z_{0}\right)$.

Assuming for the moment that we have to do with a singular point of order $k-1$, let us designate as " critical curves" that family of curves which yield the values of $f^{(j)}, g^{(j)}, h^{(j)}$ necessary to make

$$
f_{1}^{(j)}=g_{1}^{(j)}=h_{1}^{(j)}=0, \text { for } j=1,2, \cdots, k-1 .
$$

By differentiating equations (3) $j$ times we get

$$
\begin{aligned}
& f_{1}^{(j)}(t)=\varphi_{x} f^{(j)}(t)+\varphi_{y} g^{(j)}(t)+\varphi_{z} h^{(j)}(t)+\rho_{j}, \\
& g_{1}^{(j)}(t)=\psi_{x} f^{(j)}(t)+\psi_{y} g^{(j)}(t)+\psi_{z} h^{(j)}(t)+\sigma_{j}, \\
& h_{1}^{(j)}(t)=\theta_{x} f^{(j)}(t)+\theta_{y} g^{(j)}(t)+\theta_{z} h^{(j)}(t)+\tau_{j} .
\end{aligned}
$$

The functions $\rho_{j}, \sigma_{j}, \tau_{j}$ depend on the derivatives of $\varphi, \psi, \theta$ of orders $1,2, \cdots, j$, and on the derivatives of $f, g, h$ of orders $1,2, \cdots, j-1$. Then the critical curves have the directions of their corresponding transformed curves determined by $f_{1}^{(k)}\left(t_{0}\right): g_{1}^{(k)}\left(t_{0}\right): h_{1}^{(k)}\left(t_{0}\right)$. In computing these we must evaluate $\rho_{k}, \sigma_{k}, \tau_{k}$, and these depend upon the derivatives of $f, g, h$ of orders $1,2, \cdots$, $k-1$, taken at $t=t_{0}$. The values of these derivatives are determined uniquely by the equations

$$
f_{1}^{(j)}=g_{1}^{(j)}=h_{1}^{(j)}=0 \quad(j=1,2, \cdots, k-1) .
$$

For if we assume the truth of this statement for $j=1,2, \cdots, n-1(n<k)$, then $\rho_{n}, \sigma_{n}, \tau_{n}$ are constant for the family of curves in question, and the equations (30) for $j=n$ can be put in the form of (25). They are therefore independent of, and consistent with, the condition (27), for $j=n$. Therefore, our proposition is true for $j=n$. We know, however, that the equations (30) yield, for $j=1$, the values $f^{\prime}=I_{1}, g^{\prime}=I_{2}, h^{\prime}=I_{3}$, uniquely. That is, our statement is true for $j=2$; hence for $j=3$, and so on. It is, moreover, certain, that all the critical curves have with each other contact of order $k-1$ at least.

The necessary and sufficient conditions for the consistency of equations (30), considered as equations in $f^{(j)}, g^{(j)}, h^{(j)}$, are that

$$
A_{i} \rho_{j}+B_{i} \sigma_{j}+C_{i} \tau_{j}=0 \quad(j=1,2, \cdots, k-1) .
$$

If we fix our attention upon the curves which have contact of order $n-1$ $(n<k)$ with the critical curves, then the directions of the corresponding transformed curves will depend upon derivatives of order $n$ :

$$
\cos \alpha_{1}: \cos \beta_{1}: \cos \gamma_{1}=f_{1}^{(n)}: g_{1}^{(n)}: h_{1}^{(n)},
$$

where, to define $f_{1}^{(n)}, g_{1}^{(n)}, h_{1}^{(n)}$, we replace $j$ by $n$ in (29). Then, by virtue of (31),

$$
A_{i} f_{1}^{(n)}+B_{i} g_{1}^{(n)}+C_{i} h_{1}^{(n)}=A_{i} \rho_{n}+B_{i} \sigma_{n}+C_{i} \tau_{n}=0 .
$$


That is, the tangent to the transform of any one of the curves in question lies in the plane $\Pi$.

Turning now to the critical curves themselves, we see from (29) that

$$
A_{i} f_{1}^{(k)}+B_{i} g_{1}^{(k)}+C_{i} h_{1}^{(k)}=A_{i} \rho_{k}+B_{i} \sigma_{k}+C_{i} \tau_{k} .
$$

But, by (31), a sufficient condition that $\left(x_{0}, y_{0}, z_{0}\right)$ be a singular point of at least the $k$ th order, is that the right member of (32) vanish. Hence none of the critical curves can go over into a curve tangent to $I I$. If, however, using the equations (29), we give to $f_{1}^{(k)}, g_{1}^{(k)}, h_{1}^{(k)}$ a set of values consistent with (32), but otherwise arbitrary, then, (27) being enforced, we can solve uniquely for $f^{(k)}, g^{(k)}, h^{(k)}$; so that the variation of the latter quantities will yield every direction not in $\Pi$. The same argument shows that among the critical curves, those, and only those, will be transformed into tangent curves, which have contact of the $k$ th order at least. We may summarize these results as follows:

Theorem 6. At a singular point of order $k-1$, the curves whose equations do not yield the critical values of all the derivatives $f^{(j)}, g^{(j)}, h^{(j)}$ for $j=1,2, \cdots, k-1$, are transformed into curves whose tangents lie in $\Pi$; while the curves whose equations yield these critical values go over into a family of curves which among them have every direction not in $\Pi$. Among the critical curves, the condition for the preservation of tangency is contact of order $k$, at least.

We shall now generalize the result found in $\S 5$. Let us assume again, that we have to deal with a singular point of order $k-1$; and let us consider the family of critical curves. The condition that the transformed curve corresponding to one of these be tangent to the plane whose normal has the direction-ratios $\lambda: \mu: \nu$, is that

$$
\begin{aligned}
\left(\lambda \phi_{x}+\mu \psi_{x}+\nu \theta_{x}\right) & f^{(k)}+\left(\lambda \phi_{y}+\mu \psi_{y}+\nu \theta_{y}\right) g^{(k)} \\
& +\left(\lambda \phi_{z}+\mu \psi_{z}+\nu \theta_{z}\right) h^{(k)}+\lambda \rho_{k}+\mu \sigma_{k}+\nu \tau_{k}=0 .
\end{aligned}
$$

Since the plane in question is not $\Pi, \lambda: \mu: \nu \neq A_{i}: B_{i}: C_{i}$, and hence (33) is a relation of the form

$$
\xi f^{(k)}+\eta g^{(k)}+\zeta h^{(k)}+\omega=0,
$$

where

$$
I_{1} \xi+I_{2} \eta+I_{3} \zeta=0, \quad \text { but } \xi^{2}+\eta^{2}+\zeta^{2} \neq 0 \text {. }
$$

Conversely, we show exactly as for $k=2$ [see (17)] that all the curves which satisfy a particular relation of the form (34) go over into a family of curves with complanar tangents. Further, being given the relation (34), we can determine the corresponding tangent-plane by setting the coefficients in (33) respectively equal to $\xi, \eta, \zeta, \omega$, and solving for $\lambda, \mu, \nu$. There will be a set of values of the latter quantities uniquely determined by these equations, since we are assuming that $A_{i} \rho_{k}+B_{i} \sigma_{k}+C_{i} \tau_{k} \neq 0$. The equations (33) and (34) are thus entirely equivalent. 
All the curves at present under consideration have contact of order not lower than $k-1$ with each other, and hence with any surface which has contact of order as high as $k-1$ with any one of them. That is, if $F(x, y, z)=0$ is such a surface, then by hypothesis, when $t=t_{0}$,

$$
\frac{d^{i} F[f(t), g(t), h(t)]}{d t^{i}}=0 \quad(i=1, \cdots, k-1)
$$

for each critical curve. The curves which have contact of order $k$, at least, with this surface satisfy, in addition, the same condition for $i=k$, and this condition is sufficient. That is,

$$
F_{x} f^{(k)}+F_{y} g^{(k)}+F_{z} h^{(k)}+\omega_{k}=0 \text {, for } t=0 .
$$

Here $\omega_{k}$ is what $\rho_{k}$ becomes when $\phi$ is replaced by $F$. Now this is a condition of the form (34), since we mean to assume that $F_{x}, F_{y}, F_{z}$ are not all zero at $\left(x_{0}, y_{0}, z_{0}\right)$, and

$$
I_{1} F_{x}+I_{2} F_{y}+I_{3} F_{z}=0 \text {. }
$$

We are thus led to the following conclusion:

ThEOREM 7. Those members of the family of critical curves which have at $\left(x_{0}, y_{0}, z_{0}\right)$ contact of order $k$, at least, with the same surface, go over into a set of curves whose tangents are complanar; and conversely, if a set of critical curves go over into a family of curves tangent to a fixed plane, there exists a surface with which they all have contact of order $k$, at least.

Let us now alter the hypothesis, and assume that instead of $k-1$, the order of our singular point is $k$. The critical curves will now be those of the family we have just been considering, which have the critical values of $f^{(k)}, g^{(k)}, h^{(k)}$. Let us consider the curves of this same family which are no longer critical. We know that they are transformed into curves tangent to $\Pi$; and our next proposition is that those of them which have contact of at least the $k$ th order with the same surface will go over into a set of curves whose tangents either coincide, or completely fill the plane $\Pi$. The curves in question satisfy a condition of the form (35). Under the present hypothesis, however, this will not in general be of the form (33); the condition for this being that it be possible to find values of $\lambda, \mu, \nu$ which make

$$
\begin{aligned}
& \lambda \phi_{x}+\mu \psi_{x}+\nu \theta_{x}=F_{x}, \\
& \lambda \phi_{y}+\mu \psi_{y}+\nu \theta_{y}=F_{y}, \\
& \lambda \phi_{z}+\mu \psi_{z}+\nu \theta_{z}=F_{z}, \\
& \lambda \rho_{k}+\mu \sigma_{k}+\nu \tau_{k}=\omega_{k} .
\end{aligned}
$$

Since $A_{i} \rho_{k}+B_{i} \sigma_{k}+C_{i} \tau_{k}=0$, these equations are incompatible unless cer- 
tain conditions of consistency are fulfilled. These will be the same as the conditions that the equations in $f^{(k)}, g^{(k)}, h^{(k)}$,

$$
\begin{aligned}
& \phi_{x} f^{(k)}+\phi_{y} g^{(k)}+\phi_{z} h^{(k)}+\rho_{k}=0, \\
& \psi_{x} f^{(k)}+\psi_{y} g^{(k)}+\psi_{z} h^{(k)}+\sigma_{k}=0, \\
& \theta_{x} f^{(k)}+\theta_{y} g^{(k)}+\theta_{z} h^{(k)}+\tau_{k}=0, \\
& F_{x} f^{(k)}+F_{y} g^{(k)}+F_{z} h^{(k)}+\omega_{k}=0
\end{aligned}
$$

be consistent. Now, as we have seen, the first three equations admit just one set of values of $f^{(k)}, g^{(k)}, h^{(k)}$, and these characterize the critical curves. Hence the equations (36) are compatible, if, and only if, the surface $F=0$ has contact of at least the $k$ th order with the critical curves. But if they are compatible, these equations admit a one-parameter set of solutions for $\lambda, \mu, \nu$. The direction of the transformed curve is therefore confined to each of a one-parameter set of planes. These planes will, however, be found to have an axis lying in $\Pi$.

If, then, the surface $F=0$ has contact of at least the $k$ th order with the critical curves, the non-critical curves in question will go aver into tangent curves. If, however, the contact of $F=0$ with the critical curves is of order $k-1$ only, then (36) are incompatible, and (35) is not a condition of the form (33). There being no other restriction upon $f^{(k)}, g^{(k)}, h^{(k)}$, we infer that the tangents to the transformed curves will fill out completely the plane $\Pi$. The same result holds if we allow the order of the singularity to increase to any value.

But we can obtain a simpler statement of the above result. Let us fix our attention upon the family of curves which have contact of order $n$, at least, with the surface $F=0$, and whose equations yield the critical values of the derivatives $f^{(j)}, g^{(j)}, h^{(j)}$ for $j=1,2, \cdots, n-1$. This does not exclude critical curves, which, the singularity being of order $k(>n-1)$, will have, in addition, critical values up to $j=k$. If now in this family there exist curves whose equations yield the critical values of the $n$th order derivatives, then the remaining members of the family will go over into tangent curves; if no such curves exist, the tangents to the transformed curves will fill out completely the plane $\Pi$.

9. Criterion for the Order of Singularity. The conditions that $\left(x_{0}, y_{0}, z_{0}\right)$ be a singular point of order $k$, at least, are, as has been noted before, that

$$
A_{i} \rho_{j}+B_{i} \sigma_{j}+C_{i} \tau_{j}=0 \quad(j=1,2, \cdots, k) .
$$

But in order that these conditions may be intelligible, we must know more about the meaning of the symbols $\rho_{j}, \sigma_{j}, \tau_{j}$. We shall outline briefly a process 
by means of which general expressions for them may theoretically be obtained.

We know already that $\rho_{1}=\sigma_{1}=\tau_{1}=0$; and that

$$
\rho_{2}=U^{2}(\phi, I), \quad \sigma_{2}=U^{2}(\psi, I), \quad \tau_{2}=U^{2}(\theta, I) .
$$

If $k>2$, then the equations (30) for $j=1,2$ will determine uniquely [in view of 27)] certain critical values of $f^{\prime}, g^{\prime}, h^{\prime}$ and $f^{\prime \prime}, g^{\prime \prime}, h^{\prime \prime}$. These values will depend only on the values of the first and second order derivatives of $\phi, \psi, \theta$ at $\left(x_{0}, y_{0}, z_{0}\right)$ and not upon any curve or curves. The next step is to write down the third derivative, with respect to $t$, of the composite function $\phi[f(t), g(t), h(t)]$. The first three terms of this expression will be $\phi_{x} f^{\prime \prime \prime}+\phi_{y} g^{\prime \prime \prime}+\phi_{z} h^{\prime \prime \prime}$, and the remainder, after putting for the first and second derivatives of $f, g, h$ the values determined above, and in the derivatives of $\phi$

$$
x=x_{0}, \quad y=y_{0}, \quad z=z_{0},
$$

will be $\rho_{3}$. Similarly, using $\psi$ and $\theta, \sigma_{3}$ and $\tau_{3}$ are defined. The process thus begun may theoretically be continued, to yield expressions for $\rho_{k}, \sigma_{k}, \tau_{k}$. But the practical difficulties speedily become insurmountable.

We therefore find it convenient to resort to an indirect method of attacking this problem. Let us assume to begin with, that $A_{1} \neq 0$. This is in reality no restriction, for a simple substitution of symbols will suffice to achieve the same results in case this hypothesis is untenalle. Now let $\left(x_{0}, y_{0}, z_{0}\right)$, corresponding to $t=t_{0}$, be a singular point of order $k-1(k \geqq 2)$ for the transformation. Let

$$
x=f(t), \quad y=g(t), \quad z=h(t)
$$

be any curve, satisfying the general requirements laid down at the beginning, 'such that the normalization of parameter has been made, and that, if we use the notation of (3),

$$
f_{1}^{(j)}\left(t_{0}\right)=g_{1}^{(j)}\left(t_{0}\right)=h_{1}^{(j)}\left(t_{0}\right)=0 \quad(j=1,2, \cdots, k-1) .
$$

We shall also assume that if $J(t) \equiv J[f(t), g(t), h(t)]$, then

$$
J^{(j)}\left(t_{0}\right)=0 \quad(j=1,2, \cdots, k-2) .
$$

LEMMA. From the assumptions (38) and (39) it follows that

$$
A_{1} J^{(k-1)}\left(t_{0}\right)=A_{1} f_{1}^{(k)}\left(t_{0}\right)+B_{1} g_{1}^{(k)}\left(t_{0}\right)+C_{1} h_{1}^{(k)}\left(t_{0}\right) \text {. }
$$

Let us first emphasize the fact that the expression on the right-hand side is nothing but $A_{1} \rho_{k}+B_{1} \sigma_{k}+C_{1} \tau_{k}$. This becomes evident upon consultation of the definitions of $f_{1}^{(k)}, g_{1}^{(k)}, h_{1}^{(k)}$ in (29). As we have explained, $\rho_{k}, \sigma_{k}, \tau_{k}$ are determined by means of (38), so that the right member of (40) is deter- 
mined by the properties of the transformation at $\left(x_{0}, y_{0}, z_{0}\right)$, independently of the functions $f, g, h$, which are of use only for formal purposes.

Now, according to our hypothesis, we may apply Taylor's Theorem with the Remainder to the functions $f_{\cdot 1}^{\prime}, g_{1}^{\prime}, h_{1}^{\prime}$; we thus obtain the formulæ,

Further,

$$
\begin{aligned}
& f_{1}^{\prime}(t) \equiv \frac{\left(t-t_{0}\right)^{k-1}}{(k-1) !} f_{1}^{(k)}\left[t_{0}+\theta_{1}\left(t-t_{0}\right)\right], \\
& g_{1}^{\prime}(t) \equiv \frac{\left(t-t_{0}\right)^{k-1}}{(k-1) !} g_{1}^{(k)}\left[t_{0}+\theta_{2}\left(t-t_{0}\right)\right], \\
& h_{1}^{\prime}(t) \equiv \frac{\left(t-t_{0}\right)^{k-1}}{(k-1) !} h_{1}^{(k)}\left[t_{0}+\theta_{3}\left(t-t_{0}\right)\right] .
\end{aligned}
$$

$$
J(t) \equiv \frac{\left(t-t_{0}\right)^{k-1}}{(k-1) !} J^{(k-1)}\left[t_{0}+\theta_{4}\left(t-t_{0}\right)\right],
$$

where $0<\theta_{1}, \theta_{2}, \theta_{3}, \theta_{4}<1$. But, on the other hand, we have the values of $f_{1}^{\prime}, g_{1}^{\prime}, h_{1}^{\prime}$ given by (5). Therefore, in view of (41) and (42), we have,

$$
\begin{aligned}
& \frac{\left(t-t_{0}\right)^{k-1}}{(k-1) !}\left\{A_{1} f_{1}^{(k)}\left[t_{0}+\theta_{1}\left(t-t_{0}\right)\right]+B_{1} g_{1}^{(k)}\left[t_{0}+\theta_{2}\left(t-t_{0}\right)\right]\right. \\
&\left.+C_{1} h_{1}^{(k)}\left[t_{0}+\theta_{3}\left(t-t_{0}\right)\right]\right\} \equiv J(t) f^{\prime}(t) \\
& \equiv \frac{\left(t-t_{0}\right)^{k-1}}{(k-1) !} J^{(k-1)}\left[t_{0}+\theta_{4}\left(t-t_{0}\right)\right] f^{\prime}(t) .
\end{aligned}
$$

Here the partial derivatives are, as before, converted into functions of $t$.

If now in (43) we let $t$ approach $t_{0}$, we obtain,

$$
f^{\prime}\left(t_{0}\right) J^{(k-1)}\left(t_{0}\right)=A_{1} f_{1}{ }^{(k)}\left(t_{0}\right)+B_{1} g_{1}{ }^{(k)}\left(t_{0}\right)+C_{1} h_{1}{ }^{(k)}\left(t_{0}\right) \text {. }
$$

But, by hypothesis,

$$
f^{\prime}\left(t_{0}\right)=A_{1} .
$$

Therefore, the lemma stated above is true.

Let us now define functions $J_{n}(x, y, z)$, as follows:

Also, let

$$
J_{0} \equiv J, J_{1} \equiv \frac{D(J, \psi, \theta)}{D(x, y, z)}, \quad \cdots, \quad J_{n} \equiv \frac{D\left(J_{n-1}, \psi, \theta\right)}{D(x, y, z)}
$$

$$
J_{n}(t) \equiv J_{n}[f(t), g(t), h(t)] \text {. }
$$

Then it follows at once that

$$
J_{n-1}^{\prime}\left(t_{0}\right)=J_{n}\left(t_{0}\right), n=1,2, \cdots .
$$

For

$$
J_{n-1}^{\prime}\left(t_{0}\right)=\frac{\partial J_{n-1}}{\partial x} f^{\prime}\left(t_{0}\right)+\frac{\partial J_{n-1}}{\partial y} g^{\prime}\left(t_{0}\right)+\frac{\partial J_{n-1}}{\partial z} h^{\prime}\left(t_{0}\right) \text {. }
$$


But by hypothesis,

$$
f^{\prime}\left(t_{0}\right)=A_{1}, \quad g^{\prime}\left(t_{0}\right)=A_{2}, \quad h^{\prime}\left(t_{0}\right)=A_{3} .
$$

The equations (46) then result by direct citation of the definition of the functions $J_{n}$.

If we put $k=2$, the assumptions (38) entail as a necessary consequence that

$$
J\left(x_{0}, y_{0}, z_{0}\right)=0,
$$

and hence that $\boldsymbol{J}\left(t_{0}\right)=0$. Further, if $k=3$, it has already been shown that the hypotheses (38) cannot be made unless

$$
J_{1}\left(x_{0}, y_{0}, z_{0}\right)=J_{1}\left(t_{0}\right)=0
$$

hence, in view of (46), $\boldsymbol{J}^{\prime}\left(t_{0}\right)=0$. The conditions for the application of (40) are now fulfilled, and therefore,

$$
A_{1} \mathbf{J}^{\prime \prime}\left(t_{0}\right)=A_{1} f_{1}^{\prime \prime \prime}\left(t_{0}\right)+\dot{B}_{1} g_{1}^{\prime \prime \prime}\left(t_{0}\right)+C_{1} h_{1}^{\prime \prime \prime}\left(t_{0}\right) \text {. }
$$

Suppose now that the point $\left(x_{0}, y_{0}, z_{0}\right)$ is a singular point of the third order. Then among the functions $f, g, h$ for which (47) is satisfied, let us pick out a set for which, also,

$$
f_{1}^{\prime \prime \prime}\left(t_{0}\right)=g_{1}^{\prime \prime \prime}\left(t_{0}\right)=h_{1}^{\prime \prime \prime}\left(t_{0}\right)=0 .
$$

For any such set, $\boldsymbol{J}^{\prime \prime}\left(t_{0}\right)=0$. Thus the conditions are satisfied for applying (40) to the case $k=4$, and so on. We conclude, by means of the induction thus begun, that the assumptions (39) are superfluous: that is, that they are a necessary consequence of (38).

The equation (40) now yields as a necessary and sufficient condition for a singularity of order $k$ at least, that

$$
\boldsymbol{J}^{(k-1)}\left(t_{0}\right)=0 .
$$

For we have pointed out that

$$
A_{1} f_{1}^{(k)}\left(t_{0}\right)+B_{1} g_{1}^{(k)}\left(t_{0}\right)+C_{1} h_{1}^{(k)}\left(t_{0}\right)=A_{1} \rho_{k}+B_{1} \sigma_{k}+C_{1} \tau_{k} .
$$

Let us now summarize the foregoing results:

TheOREM 8. Let $\left(x_{0}, y_{0}, z_{0}\right)$ be a singular point of order at least $k-1$; and let $f(t), g(t), h(t)$ be any functions satisfying our general hypotheses (see §3) whose derivatives, $f^{(j)}, g^{(j)}, h^{(j)}$, have, for $t=t_{0}$, the critical values $(j=1,2, \cdots, k-1)$. Further, let

$$
\boldsymbol{J}(t) \equiv J[f(t), \quad g(t), \quad h(t)],
$$

and let $\boldsymbol{J}^{(j)}(t)$ represent $d^{j} \mathbf{J}(t) / d t^{j}$. Then

$$
J^{(j)}\left(t_{0}\right)=0,
$$


for $j=1,2, \cdots, k-2$; and the necessary and sufficient condition for a singularity of order $k$ at least is that also,

$$
J^{(k-1)}\left(t_{0}\right)=0 \text {. }
$$

This theorem holds, even when it is no longer true that $A_{1} \neq 0$.

Theorem 8 is of little value as a test, since, in attempting to apply it, we meet the same difficulties as were mentioned early in this paragraph. It will lead us, however, to the desired result. Let us apply the theorem to the transformation obtained by replacing $\phi$ in the original transformation by $J$. This replaces $J$ by $J_{1}$. Since

$$
J^{(j)}\left(t_{0}\right)=g_{1}^{(j)}\left(t_{0}\right)=h_{1}^{(j)}\left(t_{0}\right)=0 \quad(j=1,2, \cdots, k-2),
$$

then $J_{1}^{(j-1)}\left(t_{0}\right)=0$, for the same values of $j$. Therefore, applying (40),

where

$$
A_{1} J^{(k-2)}\left(t_{0}\right)=A_{1} J^{(k-1)}\left(t_{0}\right)+\bar{B}_{1} g^{(k-1)}\left(t_{0}\right)+\bar{C}_{1} h^{(k-1)}\left(t_{0}\right),
$$

$$
\bar{B}_{1}=\left|\begin{array}{cc}
\theta_{y} & \theta_{z} \\
J_{y} & J_{z}
\end{array}\right| \quad \text { and } \quad \bar{C}_{1}=\left|\begin{array}{ll}
J_{y} & J_{z} \\
\psi_{y} & \psi_{z}
\end{array}\right| \text {. }
$$

But $g_{1}^{(k-1)}\left(t_{0}\right)=h_{1}^{(k-1)}\left(t_{0}\right)=0$, and hence, since

$$
A_{1} J^{(k-1)}\left(t_{0}\right)=A_{1} f_{1}^{(k)}\left(t_{0}\right)+B_{1} g_{1}^{(k)}\left(t_{0}\right)+C_{1} h_{1}^{(k)}\left(t_{0}\right),
$$

we have the result,

$$
A_{1} J_{1}^{(k-2)}\left(\mathrm{t}_{0}\right)=A_{1} f_{1}^{(k)}\left(t_{0}\right)+B_{1} g_{1}^{(k)}\left(t_{0}\right)+C_{1} h_{1}^{(k)}\left(t_{0}\right) .
$$

Precisely the same reasoning shows that

$$
\begin{aligned}
J_{n}^{(k-n-1)}\left(t_{0}\right)=\cdots=J_{1}^{(k-2)}\left(t_{0}\right) & =J^{(k-1)}\left(t_{0}\right) \\
& =\frac{1}{A_{1}}\left[A_{1} f_{1}^{(k)}\left(t_{0}\right)+B_{1} g^{(k)}\left(t_{0}\right)+C_{1} h_{1}^{(k)}\left(t_{0}\right)\right] .
\end{aligned}
$$

Therefore, finally, $\boldsymbol{J}_{k-2}^{\prime}\left(t_{0}\right)=\boldsymbol{J}^{(k-1)}\left(t_{0}\right)$, or, since $\boldsymbol{J}_{n-1}^{\prime}\left(t_{0}\right)=\boldsymbol{J}_{n}\left(t_{0}\right)$,

Incidentally, it is true that

$$
J_{k-1}\left(x_{0}, y_{0}, z_{0}\right)=J_{k-1}\left(t_{0}\right)=J^{(k-1)}\left(t_{0}\right)
$$

$J\left(x_{0}, y_{0}, z_{0}\right)=J_{1}\left(x_{0}, y_{0}, z_{0}\right)=J_{2}\left(x_{0}, y_{0}, z_{0}\right)=\cdots=J_{k-2}\left(x_{0}, y_{0}, z_{0}\right)=0$.

Therefore, given a singular point of order $k-1$ at least, the necessary and sufficient condition that it be a singular point of at least the $k$ th order is that $J_{k-1}=0$ there.

We know already that when $k=1$, the condition is $J=0$; and when $k=2$, 
that it is $J=0$ and $J_{1}=0$. Hence, inductively, we arrive at the final result:

THEOREM 9 The necessary and sufficient conditions that the point $\left(x_{0}, y_{0}, z_{0}\right)$ be a singular point of order $k$, are that in that point,

$$
J=0, \quad J_{1}=0, \quad J_{2}=0, \quad \cdots, \quad J_{k-1}=0, \quad J_{k} \neq 0 . *
$$

As a by-product, we have also the following:

THEOREM 10. If the point $\left(x_{0}, y_{0}, z_{0}\right)$ is a singular point of the kth order for the transformation (1), then it is a singular point of order $k-n-1$ for the transformation

$$
x_{1}=J_{n}(x, y, z), \quad y_{1}=\psi(x, y, z), \quad z_{1}=\theta(x, y, z) .
$$

Here we rely on the assumption that $A_{1} \neq 0$. This theorem is true, however, whenever $A_{1}^{2}+A_{2}^{2}+A_{3}^{2} \neq 0$. If this last hypothesis fails, the theorem is not true as stated, but must be replaced by one of the theorems obtained by replacing $\psi, \theta$ by $\theta, \phi$ and by $\phi, \psi$ in the second transformation.

We have in equations (51), not only the desired test for the order of singularity of any point, but also the eliminants of the set of $3 k$ algebraic equations (30) ( for $j=1,2, \cdots, k$ ), considered as equations in the derivatives of $f, g, h$.

10. Order of Singularity, Geometrically Considered. Let us consider the three families of surfaces,

$$
\phi(x, y, z)=\text { const., } \quad \psi(x, y, z)=\text { const., } \theta(x, y, z)=\text { const., }
$$

which are carried by the transformation into the three families of planes parallel to the coördinate planes. The discussion of this section is carried through only in case these equations and all others of the form $F(x, y, z)=0$ which occur during the work represent surfaces (not curves or points). If we let $\phi_{0}=\phi\left(x_{0}, y_{0}, z_{0}\right)$, etc., then the surfaces

$\phi(x, y, z)-\phi_{0}=0, \quad \psi(x, y, z)-\psi_{0}=0, \quad \theta(x, y, z)-\theta_{0}=0$, shall be referred to as the $\phi$-, $\psi$-, and $\theta$-surfaces for the point $\left(x_{0} y_{0} z_{0}\right)$. Suppose, for the present, that the three surfaces are regular at $\left(x_{0}, y_{0}, z_{0}\right)$ : that is, that $\phi, \psi, \theta$ and their first derivatives are continuous there, while not all of the latter vanish; and also that they are non-tangent at this point, that is, that not all the cofactors of the elements of any row of $J$ are zero there.

Now when

$$
J\left(x_{0}, y_{0}, z_{0}\right)=0,
$$

and only then, the three tangent planes have a common line of intersection. This means that the three curves of intersection are mutually tangent. Their common direction is the critical direction, and this we should expect, since the

* For a definition of the functions $J_{i}$ see (45). 
three tangent curves are transformed into mutually perpendicular straight lines. The extension to higher singularities is immediate. Let the curve of intersection of the $\psi$ - and $\theta$-surfaces have the parametric equations,

$$
x=u_{1}(t), \quad y=v_{1}(t), \quad z=w_{1}(t) .
$$

Then we have,

$$
\psi\left[u_{1}(t), v_{1}(t), w_{1}(t)\right]-\psi_{0} \equiv 0, \quad \theta\left[u_{1}(t), v_{1}(t), w_{1}(t)\right]-\theta_{0} \equiv 0
$$

and therefore,

$$
\frac{d^{j} \psi\left[u_{1}, v_{1}, w_{1}\right]}{d t^{j}} \equiv 0, \quad \frac{d^{j} \theta\left[u_{1}, v_{1}, w_{1}\right]}{d t^{j}} \equiv 0 \quad(j=1,2, \cdots) .
$$

Similarly, using for the other curves the subscripts 2 and 3, we have,

$$
\begin{array}{ll}
\frac{d^{j} \theta\left[u_{2}, v_{2}, w_{2}\right]}{d t^{j}} \equiv 0, & \frac{d^{j} \phi\left[u_{2}, v_{2}, w_{2}\right]}{d t^{j}} \equiv 0, \\
\frac{d^{j} \phi\left[u_{3}, v_{3}, w_{3}\right]}{d t^{j}} \equiv 0, & \frac{d^{j} \psi\left[u_{3}, v_{3}, w_{3}\right]}{d t^{j}} \equiv 0
\end{array} \quad(j=1,2,3, \cdots) .
$$

Let us always suppose (27) to be in force. Then the equations (52) may be considered as linear equations which determine uniquely the values of $u_{1}^{(j)}, v_{1}^{(j)}, w_{1}^{(j)}$ up to, say, $j=k$ which belong to the corresponding curve. Similarly we use (53) and (54). If we suppose the three curves in question to have contact of order $k$ at $t=t_{0}$, then let the curve

$$
x=f(t), \quad y=g(t), \quad z=h(t)
$$

have contact of the same order with them there. The various values of the derivatives obtained from (52), (53), (54) will be equal to the values of the corresponding derivatives of $f, g, h$ for $t=t_{0}$. That is,

$$
\frac{d^{j} \phi[f, g, h]}{d t^{j}}=\frac{d^{j} \psi[f, g, h]}{d t^{j}}=\frac{d^{j} \theta[f, g, h]}{d t^{j}}=0 \quad(j=1,2, \cdots, k) .
$$

This means that the point is a singular point of the $k$ th order, at least, and the critical values of $f^{(j)}, g^{(j)}, h^{(j)}$ are yielded by each of the three curves of intersection of the $\phi-, \psi-, \theta$-surfaces. The equations (55) may be regarded as algebraic equations in the $f^{(j)}, g^{(j)}, h^{(j)}$ apart from any relation to the functions $f, g, h$. Regarding the $f^{(j)}, g^{(j)}, h^{(j)}$ as unknown quantities, and allowing the derivatives of the $u_{i}, v_{i}, w_{i}$ to play the same rôle, it is clear that the coefficients in the first two of equations (55) are the same as those of (54), and so on. The system of solutions of (55) is unique, if we assume them consistent, and is exactly the same as the system of solutions of (52), of (53), or of (54) $(j=1,2, \cdots, k)$. Therefore, if (55) are consistent, that is, if the point 
$\left(x_{0}, y_{0}, z_{0}\right)$ is a singular point of the $k$ th order, it follows that the three curves of intersection of the $\phi_{-}, \psi$-, and $\theta$-surfaces have contact of order $k$, at least, each with the others. That is:

ThEOREM 11. The necessary and sufficient condition that a given point be a singular point of order $k$, is that the three curves of intersection of the $\phi-, \psi$-, and $\theta$-surfaces for that point have with each other contact of order $k$.

If the surfaces are still regular, but two of them are tangent, then the latter are not tangent to the third. The curve of intersection of the two tangent surfaces will have a singular point, while their intersections with the third will be regular and tangent, both having the critical direction. It will always be true that $J\left(x_{0}, y_{0}, z_{0}\right)=0$, and the condition for singularity of order $k(k<1)$ will be contact of order $k$ between the two regular curves. On the other hand one (but no more than one) of the surfaces may have a singular point at $\left(x_{0}, y_{0}, z_{0}\right)$. The other two have, then, distinct tangent planes; while always there is a singularity of at least the first order. The critical values of the derivatives are yielded by the equations of the one regular curve of intersection; and the order of singularity is measured by the order of contact of this curve with the singular surface, it being understood that this contact is to be defined by the analytical conditions used at an ordinary point of the surface.

The surface $J=0$ has an interesting relation to the curves we have been considering, which we may state as follows:

THEOREM 12. A necessary and sufficient condition that $\left(x_{0}, y_{0}, z_{0}\right)$ be a singular point of order $k$ for the transformation, is that the surface $J=0$ have contact there of order $k-1$ with the curves of intersection of the $\phi_{-}, \psi_{-}$, and $\theta$-surfaces for that point.

For, as was shown in $\S 9$, if $\left(x_{0}, y_{0}, z_{0}\right)$ is a singular point of the $k$ th order and if $x=f(t), y=g(t), z=h(t)$ is a critical curve, then

$$
J^{(j)}\left(t_{0}\right)=0 \quad(j=1,2, \cdots, k-1),
$$

while the condition for a singularity of order $k+1$ is that also $\boldsymbol{J}^{(k)}\left(t_{0}\right)=0$. And these are the conditions for the contacts in question.

We also showed that, $\boldsymbol{J}_{k-1}^{(j-k+1)}\left(t_{0}\right)=\boldsymbol{J}_{k-2}^{(j-k+2)}\left(t_{0}\right)=\cdots=\boldsymbol{J}_{1}^{(j-1)}\left(t_{0}\right)=\boldsymbol{J}^{(j)}\left(t_{0}\right)=0(j=0,1,2, \cdots, k-1) ;$ where it is assumed that the equations involving a negative index are to be omitted, and that $\boldsymbol{J}_{i}^{(0)}\left(t_{0}\right)=\boldsymbol{J}_{\boldsymbol{i}}\left(t_{0}\right)$. The meaning of this we state as follows:

TheOREM 13. If the point $\left(x_{0}, y_{0}, z_{0}\right)$ is a singular point of order $k$ for the transformation, then the surface $J_{n}=0(n=0,1 \cdots k-2)$ has contact of order $k-n-1$ with the curves of intersection of the $\phi-, \psi$-, and $\theta$-surfaces. Moreover, the surface $J_{k-1}=0$ passes through the point $\left(x_{0}, y_{0}, z_{0}\right)$, and may be said to have contact of order zero with the curves in question. 
There is a rather curious version of the condition for a singularity of the second order, which does not seem to have a direct analogue in the higher cases. If we let $A_{i}, B_{i}, C_{i}$ have the values which they take on at $\left(x_{0}, y_{0}, z_{0}\right)$, then the surface

$$
A_{i}\left(\phi-\phi_{0}\right)+B_{i}\left(\psi-\psi_{0}\right)+C_{i}\left(\theta-\theta_{0}\right)=0
$$

passes through $\left(x_{0}, y_{0}, z_{0}\right)$, and, since $J=0$, it has a singular point there. If not all the second order derivatives of the left member vanish, then the tangent lines at the point in question lie on a quadric cone, whose generators have direction-ratios $l: m: n$ satisfying the relation

$$
A_{i} U^{2}(\phi, l, m, n)+B_{i} U^{2}(\psi, l, m, n)+C_{i} U^{2}(\theta, l, m, n)=0,
$$

where by $U^{2}(\phi, l, m, n)$ is meant the result of substituting $l, m, n$ for $I_{1}, I_{2}, I_{3}$ in $U^{2}(\phi, I)$. Then the equation

$$
P=A_{i} U^{2}(\phi, I)+B_{i} U^{2}(\psi, I)+C_{i} U^{2}(\theta, I)=0
$$

is the condition that the line having the critical direction be one of the generators of this cone-i.e., that it be a tangent line to the surface under consideration. This surface is carried by means of the transformation into the plane $\Pi$.

Being concerned primarily with questions of contact, we have said nothing about the behavior of the transformed curve in the neighborhood of the point $\left(x_{1}^{0}, y_{1}^{0}, z_{1}^{0}\right)$. Unless $\left(x_{0}, y_{0}, z_{0}\right)$ is a singular point of infinite order, or the functions $\phi, \psi, \theta$ lack the necessary derivatives to enable us to determine its order, then any curve whose defining functions have the requisite number of continuous derivatives goes over into a curve which is regular in the neighborhood of $\left(x_{1}^{0}, y_{1}^{0}, z_{1}^{0}\right)$. For there exists an interval on the original curve about $\left(x_{0}, y_{0}, z_{0}\right)$ such that within it, except at this point, the curve never has the critical direction. Otherwise, the functions $f_{1}^{\prime}(t), g_{1}^{\prime}(t), h_{1}^{\prime}(t)$ would vanish for all values of $t$ in a certain interval; and $f_{1}(t), g_{1}(t), h_{1}(t)$ would be constants. This would mean that the original curve had gone over into a point, and therefore that $\left(x_{0}, y_{0}, z_{0}\right)$ is a singular point of infinite order. Moreover, we have shown that the transformed curve has a definite tangent at $\left(x_{1}^{0}, y_{1}^{0}, z_{1}^{0}\right)$. Whatever peculiarities the curve has are due to the fact that, if the original curve has the critical direction, a value of $k$ exists, such that

$$
f_{1}^{(j)}\left(t_{0}\right)=g_{1}^{(j)}\left(t_{0}\right)=h_{1}^{(j)}\left(t_{0}\right)=0 \quad(j=1,2, \cdots, k-1),
$$

but $f_{1}^{(k)}, g_{1}^{(k)}, h_{1}^{(k)}$ are not all zero. Now, whenever $k$ is even, the curve will present a cusp at $\left(x_{1}^{0}, y_{1}^{0}, z_{1}^{0}\right)$; but if $k$ is odd, there will be a smooth arc through the point. In particular, the parts of the curve on opposite sides of the cusp may coincide, so that the curve appears to consist of a single branch, coming up to the point, and ending there. We make these statements briefly, 
and without proof, since we are interested only in questions of contact, for the treatment of which the present distinction is not significant.

11. Examples. The following examples, $(a)-(d)$, illustrate the foregoing theory.

$$
x_{1}=x, \quad y_{1}=y, \quad z_{1}=z^{2} .
$$

Here $J \equiv 2 z$, and $J_{1} \equiv 2$; so that all points of the plane $z=0$ are singular points of the first order, it being impossible for Case II or Case III to arise. For every point of the plane $z=0$, the critical direction is that of the perpendicular to this plane; and for each point the plane $\Pi$ is the plane $z_{1}=0$ itself. On account of the simplicity of this transformation, its properties are easily studied; and this is of considerable importance, since it is not difficult to prove that every transformation which meets the requirements of $\$ 1$, and which has in the point $\left(x_{0}, y_{0}, z_{0}\right)$ a singularity of the first order, can be expressed as the product of three transformations $R, S$, and $T$, of which $R$ and $T$ are nonsingular at $\left(x_{0}, y_{0}, z_{0}\right)$, while $S$ is the transformation given by $(a) .^{*}$

$$
\begin{array}{r}
x_{1}=x, \quad y_{1}=y, \quad z_{1}=z^{n} \quad[n \text { an integer, } \geqq 2], \\
J \equiv n z^{n-1}, \quad J_{1} \equiv n(n-1) z^{n-2}, \quad \cdots, \quad J_{n-2} \equiv n ! z, \quad J_{n-1} \equiv n !
\end{array}
$$

The points of the plane $z=0$ are all singular points of order $n-1$. There are no other singular points. The critical values for any point are furnished by the parallel to the axis of $z$ through the point, and the order of the derivatives needed to find the direction of any transformed curve will be greater by one unit than the order of the contact of the original curve with the line in question. All curves for which this order is less than $n-1$ will go over into curves tangent to the plane $z_{1}=0$. There seems to be, however, no theorem in this case analogous to that stated in $(a)$.

$$
x_{1}=2 x+z^{2}, \quad y_{1}=x+y, \quad z_{1}=z^{2}+x^{2}-2 y .
$$

Here it is allowable to set the $I$ of the general theory equal to $C$, and the $i$ equal to 3 . We then have,

$$
J \equiv-4 x z, \quad J_{1} \equiv-8\left(x-z^{2}\right), \quad J_{2} \equiv 48 z, \quad J_{3} \equiv 96 .
$$

Hence, all points of the line $x=z=0$ are singular points of the third order, while all other points of the planes $x=0$ and $z=0$ are singular points of the first order. For any singular point $\left(x_{0}, y_{0}, z_{0}\right)$, the critical direction is $-z_{0}: z_{0}: 1$, and all curves through the point, not having this direction, will be transformed into curves tangent to the plane whose normal has the directionratios $-1-x_{0}: 2: 1$ [plane $\Pi$ ] .

* A similar theorem for transformations in two dimensions was given by L. S. DEderICK, in his dissertation, deposited in the Harvard University Library in 1909. 
At the origin, a singular point of the third order, curves having contact of the first, second, and third orders with the curves of intersection of the $\phi$-, $\psi$-, $\theta$-surfaces will have the directions of their transforms determined by derivatives of the second, third, and fourth orders, respectively. For example, the line $x=y=0$ has contact of the first order with the critical curves. It is transformed into the curve, $x_{1}=z^{2}, y_{1}=0, z_{1}=z^{2}$; whose direction depends upon derivatives of the second order. This curve consists of half the line $y_{1}=0, x_{1}-z_{1}=0$, taken twice over, so that there is a cusp at the origin, although the two branches meeting there coincide. We see also that the line lies in the plane $\Pi$.

On the other hand, the curve $x=t^{3}-\frac{1}{2} t^{2}, y=t^{3}-\frac{1}{2} t^{2}, z=t$ has contact of the second order with the critical curves. It is transformed into the curve $x_{1}=2 t^{3}, y_{1}=2 t^{3}, z_{1}=t^{6}-t^{5}+\frac{1}{4} t^{4}-2 t^{3}$; whose direction depends on derivatives of the third order. It is regular at the origin, and its tangent there lies in the plane $\Pi$, whose normal has the direction-ratios $-1: 2: 1$.

(d) $\quad x_{1}=x^{2}+4 y z-2 z^{2}, \quad y_{1}=x^{2}+4 y^{2}+2 x z-z^{2}, \quad z_{1}=z^{2}$.

Here

$$
J \equiv 16 z\left(2 x y-x z-z^{2}\right) .
$$

The $J_{i}$ are complicated, and will not be written down here, but the fact is that the points of the line $x=y=z$ (except the origin) are singular points of the second order, while those of the lines $x=z=0$ and $y=z=0$ (except the origin) are of the third order. At the origin we have Case III, while all the remaining points of the plane $z=0$ and the cone $2 x y-x z-z^{2}=0$ are of the first order.

At the points of the plane $z=0$, the critical direction is given by the ratios $-8 y^{2}: 2 x y-x^{2}: 4 x y$. At the singular points of the first order in this plane, the line with this direction does not lie in the plane, but at those of the third order, on the $x$ - and $y$-axes, it is, in each case, the perpendicular, in the $x, y$ plane, to the axis containing the point. The plane $z=0$ is invariant, and is the plane $\Pi$ for each of its points.

It may be of interest to note the effect of the transformation upon the family of straight lines,

$$
y=a x+b, \quad z=0 \quad(a \neq 0, b \neq 0),
$$

all of which have at every point directions distinct from the critical direction. They are transformed into the family,

$$
x_{1}=x^{2}, \quad y_{1}=x^{2}+4(a x+b)^{2}, \quad z_{1}=0,
$$

which lie wholly in the first quadrant of the plane $z_{1}=0$. Their directions depend on derivatives of the second order, and there will be nothing peculiar about them, except at the points corresponding to the intersections of the 
curves $(\alpha)$ with the $x$ - and $y$-axes. For, given $a$ and $b$, these points will be $(0, b)$ and $(-b / a, 0)$, corresponding to $\left(0,4 b^{2}\right)$, and $\left(b^{2} / a^{2}, b^{2} / a^{2}\right)$. Moreover, the direction of the corresponding curve in $(\beta)$ will be $0: 8 a b: 0$ at $\left(0,4 b^{2}\right)$ and $-2 b / a:-2 b / a: 0$ at $\left(b^{2} / a^{2}, b^{2} / a^{2}\right)$, so that it is always tangent to the two lines $x_{1}-y_{1}=0, z_{1}=0$, and $x_{1}=z_{1}=0$, into which the $x$ - and $y$-axes are transformed. Hence the one-parameter family of $(\beta)$ which intersect at any one point of either of these lines will be tangent in that point. Indeed (cf. Theorem 1) the plane of the tangents to the corresponding curves in $(\alpha)$ contains the critical direction.

12. Outline of an Extension to the Theory. The theory as thus far developed is based essentially upon the assumption that for all curves considered,

$$
{f^{\prime}}^{2}\left(t_{0}\right)+{g^{\prime 2}}^{2}\left(t_{0}\right)+{h^{\prime}}^{2}\left(t_{0}\right) \neq 0 \text {. }
$$

This is assuredly the case of greatest interest, but we shall indicate briefly what is the nature of the problem when the above requirement is violatedwithout pretending to any degree of completeness.

Let there be taken a curve such that

$$
f^{(j)}\left(t_{0}\right)=g^{(j)}\left(t_{0}\right)=h^{(j)}\left(t_{0}\right)=0 \quad(j=1,2, \cdots, k-1),
$$

but $f^{(k)}, g^{(k)}, h^{(k)}$ are not all zero. Here, of course, $k>1$. Then, in general, the direction of the transformed curve is given by $f_{1}^{(k)}: g_{1}^{(k)}: h_{1}^{(k)}$, where we use (29) with $j=k$ and $t=t_{0}$, and set $\rho_{k}=\sigma_{k}=\tau_{k}=0$. The direction of the original curve is $f^{(k)}: g^{(k)}: h^{(k)}$. The direction of the transformed curve is thus determined, unless $J\left(x_{0}, y_{0}, z_{0}\right)=0$, and

$$
f^{(k)}: g^{(k)}: h^{(k)}=I_{1}: I_{2}: I_{3}
$$

that is, unless the point is singular, and the curve has the critical direction. Further, the curves not having the critical direction go over into curves tangent to the plane $I I$.

Now, setting $j=k+n$ and $t=t_{0}$ in (29), we have, if $n<k$,

$$
\rho_{k+n}=\sigma_{k+n}=\tau_{k+n}=0 \text {. }
$$

For the sum of the orders of the derivatives of $f, g, h$ which occur in any one term of these expressions is $k+n$ (counting each derivative as many times as it occurs). Hence any derivative of order at least as great as $k$ (unless its order be $k+n$ ) occurs in the same term with derivatives the sum of whose orders is at most $n(<k)$, and which therefore vanish for $t=t_{0}$. Moreover, the curves for which

$$
f^{(k+j)}: g^{(k+j)}: h^{(k+j)}=I_{1}: I_{2}: I_{3} \quad(j=0,1,2, \cdots, k-1)
$$

will have the directions of their corresponding transformed curves determined 
by the ratios $f_{1}^{(k+n)}: g_{1}^{(k+n)}: h_{1}^{(k+n)}$, except those for which, further,

$$
f^{(k+n)}: g^{(k+n)}: h^{(k+n)}=I_{1}: I_{2}: I_{3} \text {. }
$$

Still excepting the latter curves, then, the curves in question will go over into curves tangent to $\Pi$. If we now let $n$ run from 0 to $k-1$, we see that all curves satisfying (56), except those for which

$$
f^{(j)}: g^{(j)}: h^{(j)}=I_{1}: I_{2}: I_{3} \quad(j=k, k+1, \cdots, 2 k-1),
$$

go over into curves tangent to $\Pi$.

The transforms of the critical curves which satisfy (57) will have their directions determined, in general, by the ratios $f_{1}^{(2 k)}: g_{1}^{(2 k)}: h_{1}^{(2 k)}$ which are given by (29).

Here $\rho_{2 k}, \sigma_{2 k}, \tau_{2 k}$ depend upon the derivatives $f^{(k)}, g^{(k)}, h^{(k)}$, which we may replace by $I_{1}, I_{2}, I_{3}$. The condition that all the curves in question be disposed of by these formulæ is that $A_{i} \rho_{2 k}+B_{i} \sigma_{2 k}+C_{i} \tau_{2 k} \neq 0$. This being granted, none of our critical curves can go over into curves tangent to $\Pi$; but the directions of their transforms are otherwise not restricted. If $A_{i} \rho_{2 k}+B_{i} \sigma_{2 k}+C_{i} \tau_{2 k}=0$, we have a singularity of a higher order, and those curves of the family under consideration, which do not yield the critical values of $f^{(2 k)}, g^{(2 k)}, h^{(2 k)}$ go over into curves tangent to $\Pi$.

Continuing, we note that the quantities $\rho_{2 k+n}, \sigma_{2 k+n}, \tau_{2 k+n}$ depend upon the derivatives of $f, g, h$ of orders $k, k+1, \cdots, k+n$, so that not until $n=k$ do we need to make use of the critical values of $f^{(2 k)}, g^{(2 k)}, h^{(2 k)}$. We may use as the critical values up to $n=k-1$,

$$
f^{(j)}=I_{1}, \quad g^{(j)}=I_{2}, \quad h^{(j)}=I_{3} \quad(j=k, k+1, \cdots, k+n) .
$$

After this, the critical values are given by the equations (see (29))

$$
f_{1}^{(2 k+j)}=g_{1}^{(2 k+j)}=h_{1}^{(2 k+j)}=0 \quad(j=0,1,2, \cdots, n-1) .
$$

We must suppose, in order that these equations may be consistent, that

$$
A_{i} \rho_{2 k+j}+B_{i} \sigma_{2 k+j}+C_{i} \tau_{2 k+j}=0 \text {. }
$$

If (58) is not satisfied for $j=n$, then the curves having equations which furnish the critical values of the derivatives thus far determined will have the directions of their transforms given by the ratios $f_{1}^{(2 k+n)}: g_{1}^{(2 k+n)}: h_{1}^{(2 k+n)}$, these quantities being defined by replacing, in (29), $j$ by $2 k+n$. All other curves will go over into curves tangent to $\Pi$, whereas none of the critical curves in question have this property, although, as before, their transforms will take every direction not in this plane.

The conditions (58) form the tests for the order of a singular point, but their use involves the solution of a system of linear equations. We might define 
the order of a singular point as in the previous case, but the classification would be unsatisfying, since it would depend not only upon the properties of the transformation but also upon the value of $k$. The curves we have been considering in this section do not often occur, and in many cases a change of parameter will remove the peculiarities in their equations.

13. Transformation of Surfaces. Let us now seek to determine the effect of the transformation upon the contact of surfaces at a singular point. In this connection there will be the special problem of determining the tangent plane to the transform of a given surface at a point corresponding to a singular point. The usual method of "extending" the transformation is inadequate when the Jacobian becomes equal to zero. Let the surface to be transformed have the equation $F(x, y, z)=0$, where $F(x, y, z)$ is continuous at $\left(x_{0}, y_{0}, z_{0}\right)$ and has all its partial derivatives of order less than or equal to that of the derivative of highest order which is used explicitly, also continuous there. It is further assumed that not all the first derivatives vanish at $\left(x_{0}, y_{0}, z_{0}\right)$.

The method of procedure will be to transform the surface by transforming all the regular arcs passing through the point $\left(x_{0}, y_{0}, z_{0}\right)$ which lie upon it. For this the way has been prepared in what has gone before. To begin with, if the surface is not tangent to the critical curves, then every regular curve upon it which passes through $\left(x_{0}, y_{0}, z_{0}\right)$ will have a direction distinct from the critical direction, and will therefore go over into a curve tangent to the plane II. No two of these curves which are non-tangent can be transformed into tangent curves, since the plane of their tangents does not contain the critical direction. The directions of the transformed curves completely fill out the plane $\Pi$, which will be the tangent plane to the transformed surface. This is the general case.

On the other hand, if the surface is tangent to the critical curves, then (assuming for the present that the singular point is of the first order) we know that the family of curves in question will in general go over into tangent curves; for they have a common tangent plane through the critical tangent. But those curves of the family which are also critical curves will go over into a set of curves with a common tangent plane. We are, moreover, in a position to compute the direction of the normal to this plane. The details will be reserved for the general case.

Let $\left(x_{0}, y_{0}, z_{0}\right)$ be a singular point of order $k-1$, and let the surface $F=0$ have contact of order at least $k-1$ with the critical curves. Then every regular curve lying upon $F=0$, passing through $\left(x_{0}, y_{0}, z_{0}\right)$, but not having contact of order as high as $k-1$ with the critical curves, will go over into a curve tangent to a fixed line $L$ which lies in the plane II (see $\S 8$ ). The curves which have contact of order $k-1$, at least, with the critical curves, will go over into a family of curves having a common tangent plane through $L$, 
whose normal has the direction-ratios $\lambda: \mu: \nu$; these numbers being determined by the equations (36). We may take as the conditions for the tangency of surfaces that the corresponding pairs of first derivatives of the left members of their equations be equal. It is clear, then, that among the surfaces satisfying the requirements of this paragraph, those, and only those, will be transformed into tangent surfaces which are tangent to each other and for which the quantity $\omega_{k}$ (see (35)) has the same value. This latter condition is the same as that every curve with the critical direction which has contact of order as high as $k-1$ with one of the surfaces shall have contact of at least this order with the others also. Moreover, it follows, from what was said above, that the family of surfaces tangent to a given surface will go over into a family whose tangent planes form a pencil about a fixed axis in the plane $\Pi$.

But if we now alter the hypothesis, and let $\left(x_{0}, y_{0}, z_{0}\right)$ be a singular point of order $k$, then the developments of $\S 8$ show that two alternatives confront us. If $F=0$ has contact of order $k$, or higher, with the critical curves, then we need only, in the above discussion, change $k-1$ into $k$. But if $F=0$ has not contact of order as high as $k$ with the critical curves, it is transformed into a surface tangent to $I I$.

Our assumption concerning $F$ is this: there exists a value $n$, such that all the derivatives of $F$, up to and including those of order $n+1$, are continuous at $\left(x_{0}, y_{0}, z_{0}\right)$, and that, if $x=f(t), y=g(t), z=h(t)$ is a critical curve, $d^{i} F(f, g, h) / d t^{i}$ vanishes when $t=t_{0}$ for $i=1, \cdots, n$ but does not vanish when $i=n+1$. Assuming that $F$ is such a function, we state our result as follows:

TheORem 14. Let $\left(x_{0}, y_{0}, z_{0}\right)$ be a singular point of order $k$. Then, if the surface $F=0$ has contact there of order not less than $k$ with the critical curves, the normal to the surface into which it is transformed will have at $\left(x_{1}^{0}, y_{1}^{0}, z_{1}^{0}\right)$ the direction-ratios $\lambda: \mu: \nu$, these numbers being obtained from equations (36) with $k$ changed to $k+1$. But if $F=0$ does not have contact of order as great as $k$ with the critical curves, it is transformed into a surface having II as its tangent plane.

What has been said above seems contradictory; for if we pick out a curve through $\left(x_{0}, y_{0}, z_{0}\right)$ which goes over into a smooth curve through $\left(x_{1}^{0}, y_{1}^{0}, z_{1}^{0}\right)$, and pass two surfaces through it, the surfaces will in general be transformed into tangent surfaces whose curve of intersection has a multiple-point at $\left(x_{1}^{0}, y_{1}^{0}, z_{1}^{0}\right)$. A simple example will show how this difficulty may be removed. Let us transform the straight line $x=y=z$ by means of the transformation $x_{1}=x, y_{1}=y, z_{1}=z^{2}$. The transformation is regular at all points of this curve, except the origin. The curve goes over into,

$$
x_{1}=t, \quad y_{1}=t, \quad z_{1}=t^{2},
$$

which is tangent to the plane $z_{1}=0$, and is, besides, perfectly regular, at the 
origin. Now the two planes, $x-z=0$ and $y-z=0$, may be used to determine the original curve. They go. over into the pair of parabolic cylinders

$$
z_{1}=x_{1}^{2} \quad \text { and } \quad z_{1}=y_{1}^{2},
$$

which are tangent to the plane $z_{1}=0$ at the origin. Their complete curve of intersection has there a double-point. But this curve consists of two branches: one in the plane $x_{1}-y_{1}=0$, and the other in the plane $x_{1}+y_{1}=0$. The first is the curve (59), while the second is the curve

$$
x_{1}=t, \quad y_{1}=-t, \quad z_{1}=t^{2},
$$

which corresponds to the straight line $x=-y=z$. The latter is determined by the planes $x-z=0$, and $y+z=0$, which by means of the given transformation, also are carried into the pair of parabolic cylinders (60).

To illustrate the process described above, let us consider the transformation

$$
x_{1}=2 x+z^{2}, \quad y_{1}=x+y, \quad z_{1}=z^{2}+x^{2}-2 y,
$$

which has been briefly discussed in Example (c) of $\S 11$. The origin is, for this transformation, a singular point of the third order, and the surface

$$
F(x, y, z) \equiv x^{2}+y^{2}+x y+x+y=0
$$

has there contact of the third order with the critical curves. Hence the transform of $F=0$ will have, at the origin, a normal whose direction-ratios are $\lambda: \mu: \nu$, determined by means of equations (36) for $k=4$ [see Theorem 14]. The intersection of the $\phi$ - and $\psi$-surfaces, $2 x+z^{2}=0, x+y=0$, is a critical curve. The parametric equations are,

Then

$$
x=f(t) \equiv-\frac{1}{2} t^{2}, \quad y=g(t) \equiv \frac{1}{2} t^{2}, \quad z=h(t) \equiv t .
$$

$$
\rho_{4}=\left[\frac{d^{4} \phi[f, g, h]}{d t^{4}}-\phi_{x} f^{\mathrm{iv}}-\phi_{y} g^{\mathrm{iv}}-\phi_{z} h^{\mathrm{iv}}\right]_{t=0}=0 .
$$

Similarly, using $\psi, \theta, F$ successively in place of $\phi$, we obtain $\sigma_{4}=0, \tau_{4}=6$, $\omega_{4}=6$. Therefore, the equations (36) become,

whence

$$
2 \lambda+\mu=1, \quad \mu-2 \nu=1, \quad 6 \nu=6,
$$

$$
\lambda=-1, \quad \mu=3, \quad \nu=1 .
$$

This result will be verified if we actually transform the surface $F=0$, obtaining the surface

$$
G \equiv y_{1}^{2}+3 y_{1}-x_{1}+z_{1} \mp y_{1} \sqrt{2 y_{1}-x_{1}+z_{1}}=0 .
$$

The direction-ratios of the normal will be given by $G_{x_{1}}: G_{y_{1}}: G_{x_{1}}$ or

$$
-1 \pm \frac{y_{1}}{\sqrt{2 y_{1}-x_{1}+z_{1}}}: 3+2 y_{1} \pm \frac{x_{1}-y_{1}-z_{1}}{\sqrt{2 y_{1}-x_{1}+z_{1}}}: 1 \mp \frac{y_{1}}{2 \sqrt{2 y_{1}-x_{1}+z_{1}}} .
$$


These derivatives become infinite at $(0,0,0)$, but their ratios have the limits $-1: 3: 1$.

14. Note on Cases II and III. In these cases no such definite and simple classification of singularities is possible as in Case $I$. If the definition of the order of singularity already given is retained, there will be a wide variation in the behavior of the transformation at different points of the same order, which in a complete discussion must be accounted for. The fundamental distinction is that the critical values are not uniquely determined at any stage. Therefore, in discussing the compatibility of the equations

$$
f_{1}^{(j)}=g_{1}^{(j)}=h_{1}^{(j)}=0 \quad(j=1,2, \cdots, k),
$$

we must consider that they all contain the derivatives $f^{(j)}, g^{(j)}, h^{(j)}$ as $u n$ known quantities. These equations are not therefore to be treated as equations of the first degree, as in Case I, and the problem of elimination presented is probably too formidable for complete solution. We do not look, then, in these cases, for such explicit analytical conditions as those of Theorem 9 . In Case II, I have been able to determine these conditions for $k=2$, and also, except for the conditions mentioned, to discuss completely the transformation of curves. It is rather remarkable that the various sub-cases which arise under Case III present the same problems as Case I, Case II, and Case III itself, so that each sub-case is practically as large a problem as the original one. The discussion here has been carried out with less detail, but the essential outlines of the theory have been constructed. These results I hope to amplify, and perhaps to present in a later naper.

Miami University, OxFord, OHIO, December, 1911. 